\title{
NONNEGATIVELY CURVED ALEXANDROV SPACES WITH SOULS OF CODIMENSION TWO
}

\author{
XUEPING LI
}

\begin{abstract}
In this paper, we study a complete noncompact nonnegatively curved Alexandrov space $A$ with a soul $S$ of codimension two. We establish some structural results under additional regularity assumptions. As an application, we conclude that in this case Sharafutdinov retraction, $\pi: A \rightarrow S$, is a submetry.
\end{abstract}

\section{INTRODUCTION}

We begin with the classical Soul Theorem of Cheeger-Gromoll ([10]) on complete noncompact Riemannian manifolds of nonnegative sectional curvature:

Theorem 0.1. Let $M$ be a complete noncompact Riemannian n-manifold with sectional curvature $\sec (M) \geqslant 0$. Then $M$ contains a compact totally geodesic submanifold $S$ (called a soul of $M$ ) such that $M$ is diffeomorphic to the normal bundle of $S$.

When $\sec (M)>0$, Gromoll and Meyer (15) earlier showed that a soul is a point, and thus $M$ is diffeomorphic to $\mathbb{R}^{n}$. Cheeger and Gromoll proposed the following so-called Soul Conjecture: If a complete noncompact nonnegatively curved Riemannian manifold has strictly positive sectional curvature around a point, then a soul is a point.

In 1994, Perelman ([26]) proved the following theorem, which implies the Soul Conjecture:

Theorem 0.2. Let $M$ be a complete noncompact Riemannian n-manifold with $\sec (M) \geqslant 0$, and let $S$ be a soul. If $P: M \rightarrow S$ is a distance nonincreasing map, then the following properties hold:

(0.2.1) For any $x \in S$ and any unit vector $v$ at $x$ normal to $S, P\left(\exp _{x}(t v)\right)=$ $x$, for all $t \geqslant 0$.

(0.2.2) Let $\gamma:[0, l] \rightarrow S$ be a geodesic, and let $V(s)$ denote the parallel vector field along $\gamma(s)$ with $V(0)=v$. Then $\sigma_{s}(t)=\exp _{\gamma(s)}(t V(s))$ are geodesics filling a flat totally geodesic strip $(t \geqslant 0)$. If $\gamma([0, l])$ is minimal, then $\left.\sigma_{s}\left(t_{0}\right)\right|_{[0, l]}$ are minimal for any fixed $t_{0}$.

(0.2.3) $P$ is a $C^{1}$-Riemannian submersion such that the eigenvalue of the second fundamental form of $P$-fibers are bounded above by the inverse of the injectivity radius of $S$ (in the barrier sense).

Received by the editors December 31, 2012.

2010 Mathematics Subject Classification. Primary 53C20, 53C99; Secondary 57N65.

Key words and phrases. Alexandrov space, soul, Sharafutdinov retraction, submetry, topologically nice, space of directions, join. 
Note that (0.2.2) implies that if $S$ is not a point, then any point in $M$ is on some flat totally geodesic strip, and thus the Soul Conjecture.

Note that it was shown independently by Cao-Shaw ([12]) and Wilking ([39]) that $P: M \rightarrow S$ is actually smooth (cf. [17]).

In this paper, we are concerned with the analogue of Theorem 0.2 in Alexandrov geometry. An Alexandrov space is a complete length space on which Toponogov's triangle comparison holds. The study of Alexandrov spaces was initiated by BuragoGromov-Perelman ([3]), partially motivated by the fact that the Gromov-Hausdorff limit of a sequence of Riemannian manifolds with sectional curvature bounded from below uniformly is an Alexandrov space which in general may have both geometric and topological singularities.

In [24, 6.3], Perelman extended Theorem 0.1 to Alexandrov spaces:

Theorem 0.3. Let $A$ be a complete noncompact nonnegatively curved Alexandrov space. Then there is a compact convex subset $S$ (called a soul of $A$ ) without boundary and a distance nonincreasing deformation retraction $\pi: A \rightarrow S$.

The map $\pi$ is called the Sharafutdinov retraction of $A$. In further study of Theorem 0.3 , there are two basic questions:

\section{Open Problem 0.4.}

(0.4.1) (26]). Soul Conjecture: If a complete noncompact nonnegatively curved Alexandrov space has strictly positive curvature around a point, then a soul is a point.

(0.4.2) ([1, 18.5]). Is the Sharafutdinov retraction $\pi$ a submetry? (A submetry is a map which preserves all $r$-balls, and thus submetry is a metric analogue of Riemannian submersion.)

An affirmative answer to (0.4.2) will easily imply (0.4.1) (see the proof of Corollary 0.6 ), but the converse may not be true.

(In the following context, when we say a minimal geodesic from one point to a subset, we always mean one whose length realizes the distance from the point to the subset.)

In [42, 2.1], Yamaguchi partially generalized (0.2.2):

Theorem 0.5. Let $A$ be a complete noncompact nonnegatively curved Alexandrov space. Let $C$ be a convex closed subset of $A$, with boundary $\partial C \neq \emptyset$, let $f=$ dist $_{\partial C}$ and let $\gamma(t) \subset C(t \in[0, b])$ be a minimal geodesic with $\gamma(0)=p, \gamma(b)=$ $q$, such that $f(\gamma(t))=$ const. Then for any minimal geodesic $\gamma_{0}$ from $p$ to $\partial C$, with $\angle\left(\gamma_{0}^{+}(0), \gamma^{+}(0)\right)=\frac{\pi}{2}$, there is a minimal geodesic $\gamma_{1}$ from $q$ to $\partial C$ such that $\left\{\gamma, \gamma_{0}, \gamma_{1}\right\}$ bounds a flat totally geodesic rectangle.

As seen earlier in the Riemannian case, (0.2.2) implies the Soul Conjecture. In comparison, a gap between Theorem 0.5 and (0.4.1) is that for a complete noncompact nonnegatively curved Alexandrov space, there may be points where no flat totally geodesic rectangle obtained in Theorem 0.5 passes through. However in the case that $\operatorname{codim}(S)=1$ (cf. 37]), Theorem 0.5 implies an affirmative answer to (0.4.2).

In this paper, we will investigate the structure of a complete noncompact nonnegatively curved Alexandrov space which is topologically nice and whose soul has codimension 2. A point in an Alexandrov space is called topologically nice if the iterated spaces of directions are all homeomorphic to spheres. An Alexandrov 
space is called topologically nice if all points on it are topologically nice. The limit space of a sequence of noncollapsed Riemannian manifolds with sectional curvature bounded from below uniformly is topologically nice.

We now begin to state the main results in this paper.

Theorem A. Let $A$ be a complete noncompact nonnegatively curved Alexandrov space. Let $S$ be a soul of $A$ and let $\pi: A \rightarrow S$ be the Sharafutdinov retraction. Suppose that $A$ is topologically nice and that $S$ is of codimension 2 . Then $\pi$ is a submetry.

We point out that the regularity assumption in Theorem A is used to classify the space of directions of points on $S$, which is crucial in the proof of Theorem A. Using Theorem A, one easily gets an affirmative answer to Open Problem (0.4.1) in the following 4-dimensional topological manifold case.

Corollary 0.6. Let $A$ be a complete noncompact nonnegatively curved 4-dimensional Alexandrov space. Suppose $A$ is a topological manifold. If $A$ has positive curvature around a point, then a soul is a point.

We now explain the main ideas in the proof of Theorem A. We may assume that $A$ is simply connected. (If $A$ is not simply connected, one can pass to the universal cover; see Lemma 1.5.)

For $p \in S$ and $v \in \Uparrow_{p}^{\partial \Omega_{c}}$ (all directions at $p$ of minimizing geodesics from $p$ to $\partial \Omega_{c}$ ), where $c$ is a fixed noncritical value of the Busemann function $f$ (defined in Section 1.1) and $\partial \Omega_{c}=f^{-1}(c)$, there is always a ray $\sigma$ at $p$ such that $\sigma^{+}(0)=v$. We call such ray a special normal ray to $S$. Let $\mathscr{F} \subseteq A$ be the union of points on all such rays:

$$
\mathscr{F}=\left\{x \in A \mid x \in \sigma: \text { a ray with } \sigma(0)=p \in S, \sigma^{+}(0)=v \in \Uparrow_{p}^{\partial \Omega_{c}}\right\} .
$$

Observe that in the special case $\mathscr{F}=A$, Theorem A follows easily from Theorem 0.5 (see the proof following Lemma 1.1).

If $\mathscr{F} \neq A$, we set

$F_{v}=\bigcup\{x \mid x \in$ flat totally geodesic strips in $A$ spanned by $\sigma$ and all minimal geodesics in $S$ from $p$ to all the points in $S$ \}.

We have the following:

Key Lemma 0.7. Let the assumptions be as in Theorem $A$, and assume that $A$ is simply connected. If $\mathscr{F} \neq A$, then for $v \in \Uparrow_{p}^{\partial \Omega_{c}}, F_{v}$ with the restricted metric isometrically splits, i.e., $F_{v} \stackrel{\text { isom }}{\cong} S \times \mathbb{R}_{+}^{1}$.

Our proof of Key Lemma 0.7 relies on a property of space of directions on $S$ (Proposition 2.1; note that if we can solve Conjecture 4.5 completely for the case in Theorem A, then the proof of Theorem A is a little simpler), where the regularity conditions are required. Assuming Key Lemma 0.7, we can choose $F=$ $\bigcup_{1 \leq i \leq l} F_{v_{i}}, l \leq 3$, such that the distance function from $F$, $\operatorname{dist}_{F}$, is concave in $A \backslash F$ (see Lemma 2.8). For any given point $x \in A \backslash F$, let $\hat{x} \in(S, a) \subset F_{u} \subset F$ such that $|x \hat{x}|=|x F|$. When $a \neq 0$, using the concavity of $\operatorname{dist}_{F}$, we can construct a "gradient flow" of $\operatorname{dist}_{F}$ from $(S, a)$ passing $x$, denoted by $\Psi_{a}^{t}$, which is distance nonincreasing (cf. 30]). 
Consider the composition $i \circ \pi \circ \Psi_{a}^{t}:(S, a) \rightarrow(S, a)$ (where $i: S \rightarrow(S, a)$ is the natural isometry), which is distance nonincreasing and a deformation, thus onto since $t=0$ is onto. A standard argument shows that $\left.\pi\right|_{\Psi_{a}^{|x \hat{x}|}((S, a))}$ is an isometry. We denote $\Psi_{a}^{|x \hat{x}|}((S, a))$ by $S_{x}$. When $a=0$, we use a limit argument (see 2.4$)$ to get an $S_{x} \ni x$ such that $\left.\pi\right|_{S_{x}}$ is an isometry.

With the above preparations, we are ready to explain that the Sharafutdinov retraction $\pi: A \rightarrow S$ is a submetry. First $\pi$ is distance nonincreasing (Theorem $0.3)$. For any $\bar{y} \in S$, it suffices to find $y \in A$ such that $|x y|=|\pi(x) \bar{y}|$ and $\pi(y)=\bar{y}$. Now it is clear that $y=S_{x} \cap \pi^{-1}(\bar{y})$ satisfies the desired condition.

Our argument can be viewed as a generalization of [37, where noncompact nonnegatively curved Alexandrov spaces with souls of codimension 1 are considered.

We organize the rest of the paper as follows:

In Section 1, we will collect some basic notions and properties which will be used throughout the paper.

In Section 2, we will prove Theorem A by assuming Proposition 2.1.

In Section 3, some applications are proved.

In Section 4, we will prove some structural results for spaces of directions of points on $S$ and verify Proposition 2.1 at the end.

\section{Preliminaries}

We start this section by fixing some notation:

$\operatorname{dist}_{x}(y)=|x y|: \quad$ the distance between points $x, y \in A$

$\operatorname{Alex}^{n}(\kappa)$ : the class of complete n-dimensional Alexandrov spaces with curvature $\geq \kappa$

$\partial A$ : the boundary of $\mathrm{A}, A \in \operatorname{Alex}^{m}(\kappa)$

$S^{n}(\kappa)$ : the $n$-space form of curvature $\kappa$

$B(p, r)=\{x|| x p \mid \leq r\}$

$S(p, r)=\{x|| x p \mid=r\}$

$\operatorname{Fr}(C)$ : the union of points where any neighborhood contains points in $C$ and in the complement of $C$

$[p q]: \quad$ a minimal geodesic from $p$ to $q, p, q \in A$

$\uparrow_{p}^{q}$ : a direction at $p$ of a minimizing geodesic from $p$ to $q$

$\Uparrow_{p}^{q}$ : $\quad$ the set of all directions at $p$ of minimizing geodesics from $p$ to $q$

$\angle\left(\uparrow_{y}^{x}, \uparrow_{y}^{z}\right): \quad$ the angle between $\uparrow_{y}^{x}$ and $\uparrow_{y}^{z}$

$\tilde{L}_{k}(x, y, z): \quad$ the corresponding comparison angle on space form $S_{2}^{k}$

$[C D]=\{x \mid x \in[c d], c \in C, d \in D\}$, where $C, D$ are subsets of $A$

$C^{\perp}=\left\{v \in A|| v C \mid=\frac{\pi}{2}\right\}$, where $C \subset A$

Flat totally geodesic strip $P$ in $X, X \in$ Alex: $\quad P$ is the image of an isometric embedding from $\left\{(x, y) \in \mathbb{R}^{2} \mid 0 \leq x \leq 1, y \geq 0\right\}$ with the standard flat metric to $X$.

For basic notions related to Alexandrov spaces, we refer to [2], 3], 29], 31] and 35 .

In the following, we shall briefly recall the construction of souls using the Busemann function and the construction of the Sharafutdinov retractions. Then we shall establish some properties which will be used in our proof or which may not be found in the literature. 
1.1. Souls and Sharafutdinov retractions. Throughout this paper, we call a subset $C$ convex if for any $p, q$ in $C, C$ contains at least one minimal geodesic joining $p, q$.

Let $A \in \operatorname{Alex}^{n}(0)$ be noncompact, and let $p \in A$. The Busemann function at $p$ is defined by

$$
f(x)=\lim _{t \rightarrow \infty}(|x, S(p, t)|-t),
$$

and $f$ is a proper concave function with definite maximum $a_{0}=\max _{x \in A}\{f(x)\}$. Then $C_{0}=f^{-1}\left(a_{0}\right)$ satisfies that for any two points, all minimal geodesics joining them are contained in $C_{0}$ (and thus $C_{0}$ is convex). If $\partial C_{0}=\emptyset$, then $C_{0}=S$, a soul of $A$. Otherwise, the distance function, $f_{1}=\operatorname{dist}_{\partial C_{0}}: C_{0} \rightarrow \mathbb{R}^{1}$, is again concave. Let $a_{1}=\max _{x \in C_{0}}\left\{f_{1}(x)\right\}$, and let $C_{1}=f_{1}^{-1}\left(a_{1}\right)$. Repeating the above process for $C_{1}$ and after a finite number of steps, we obtain $C_{k}=S$, a convex subset without boundary.

Next we will recall the construction of a distance nonincreasing deformation retraction from $A$ to $S$, the so-called Sharafutdinov retraction.

Let $\nabla_{q} f$ denote the gradient of $f$ at $q$. Since $f$ is concave, there are $f$-gradient curves. We reparameterize gradient curves so that a new curve $\alpha(t) \subset\left(A-C_{0}\right)$ satisfies that $\alpha(0)=x$ and $\alpha^{+}(t)=\frac{\nabla_{\alpha(t)} f}{\left|\nabla_{\alpha(t)} f\right|^{2}}$. Let $\beta(t) \subset\left(A-C_{0}\right)$ be the reparametrization of the gradient curve with $\beta(0)=y$. Without loss of generality, we may assume that $f(x) \leq f(y)$ and $f\left(\alpha\left(t_{0}\right)\right)=f(y)$. By a direct computation, we get

$$
|\alpha(t) \beta(0)|^{+}(t)=-\left\langle\alpha^{+}(t), \Uparrow_{\alpha(t)}^{\beta(0)}\right\rangle \leq 0, t \leq t_{0}
$$

Hence

$$
\left|\alpha\left(t_{0}\right) \beta(0)\right| \leq|\alpha(0) \beta(0)|
$$

and

$$
\left|\alpha\left(t+t_{0}\right) \beta(t)\right|^{+}(t)=-\left\langle\alpha^{+}\left(t+t_{0}\right), \Uparrow_{\alpha\left(t+t_{0}\right)}^{\beta(t)}\right\rangle-\left\langle\beta^{+}(t), \Uparrow_{\beta(t)}^{\alpha\left(t+t_{0}\right)}\right\rangle \leq 0 .
$$

Therefore $\left|\alpha\left(t+t_{0}\right) \beta(t)\right| \leq|\alpha(0) \beta(0)|$. From this we can get that $\alpha(t)$ can be uniquely extended to include the points on $C_{0}$, denoted by $\bar{\alpha}(t)$. Define a map, $\pi_{0}: A \rightarrow C_{0}$, by $\pi_{0}(x)=\bar{\alpha}\left(a_{0}-f(x)\right)$, with $x=\bar{\alpha}(0)$. We have showed that $\pi_{0}$ is distance nonincreasing.

If $\partial C_{0} \neq \emptyset$, repeating the above, we obtain that $\pi_{1}: C_{0} \rightarrow C_{1}$ is distance nonincreasing. Eventually, we will get the Sharafutdinov retraction $\pi=\pi_{k} \circ \cdots \circ \pi_{0}$.

1.2. Flat totally geodesic strips. The goal of this subsection is to give the following unbounded version of Theorem 0.5, which is known to experts (42]). Since we cannot find a complete proof in the literature, for the convenience of readers, we include a proof here.

A useful alternative expression of $f$ is: for any $c<a_{0}=\max _{x \in A}\{f(x)\}$, for $x \in$ $\Omega_{c}=f^{-1}\left(\left[c, a_{0}\right]\right), f(x)=\left|x \partial \Omega_{c}\right|+c$ [7, Proposition A.1 (5)]).

Lemma 1.1. Let $A \in \operatorname{Alex}^{n}(0)$ be noncompact, and let $f$ be a Busemann function. Then the following properties hold:

(1.1.1) For $p \in S$, let $q \in \partial \Omega_{f(q)}$ be such that $|p q|=\left|p \partial \Omega_{f(q)}\right|$. Then $[p q]$ can be extended to a ray $\gamma$, with $\gamma(0)=p$ and $|p \gamma(t)|=\left|p \partial \Omega_{f(\gamma(t))}\right|$, for any $t \geq 0$.

(1.1.2) For $p \neq r \in S$, there exists a ray $\sigma$ with $\sigma(0)=r$ and $|r \sigma(t)|=\left|r \partial \Omega_{f(\sigma(t))}\right|$, for any $t \geq 0$, and $\{\gamma,[p r], \sigma\}$ bounds a flat totally geodesic strip. 
Proof of Theorem A for the case that $\mathscr{F}=A$. For any $x \in A$, we have that $x \in \gamma$ : a special normal ray from $\bar{x}$. Hence $\pi(x)=\bar{x}$ (see Lemma (1.1.1)). For any $\bar{y} \in S$, by Lemma (1.1.2), there is a flat totally geodesic strip determined by $\{\gamma,[\bar{x} \bar{y}]\}$ in which we can find $y \in \pi^{-1}(\bar{y})$ such that $|\bar{x} \bar{y}|=|x y|$.

In the proof of Lemma 1.1 we will use the following lemma.

Lemma $1.2([42,2.5])$. Let $\Sigma \in \operatorname{Alex}^{n}(1)$, and let $C \subset \Sigma$ be a locally convex closed subset without boundary with positive dimension. If $v \in \Sigma$ such that $|v C| \geq \frac{\pi}{2}$, then $|v \xi|=\frac{\pi}{2}$, for any $\xi \in C$.

We emphasize that Lemma 1.2 will frequently be used throughout the paper.

Let $X$ be an Alexandrov space. For $p \in X$, let $T_{p} X$ (or $T_{p}$ ) denote the tangent cone of $X$ at $p$, and let $\Sigma_{p} X$ (or $\Sigma_{p}$ ) denote the space of directions of $X$ at $p$.

Proof of Lemma 1.1. (1.1.1): Let $q_{1} \in A$ such that $f\left(q_{1}\right)<f(q)$ and $\left|q q_{1}\right|=$ $\left|q \partial \Omega_{f\left(q_{1}\right)}\right|$. Then $f(p)-f\left(q_{1}\right)=\left|p \partial \Omega_{f(q)}\right|+\left|q \partial \Omega_{f\left(q_{1}\right)}\right| \geq|p q|+\left|q q_{1}\right| \geq\left|p q_{1}\right| \geq$ $\left|p \partial \Omega_{f\left(q_{1}\right)}\right|=f(p)-f\left(q_{1}\right)$. Thus $[p q] \cup\left[q q_{1}\right]$ is a minimal geodesic with the desired property. Iterating this process, one can get the desired ray $\gamma(t)$.

(1.1.2): Note that $|p q|=\left|p \partial \Omega_{f(q)}\right|$ implies that $|p q|=|q S|$. Then by the first variation formula, $\left|\uparrow_{p}^{q} \Sigma_{p} S\right| \geq \frac{\pi}{2}$, and by Lemma $1.2,\left|\uparrow_{p}^{q} v\right|=\frac{\pi}{2}$, for any $v \in \Sigma_{p} S$. Thus by Theorem 0.5 , for $t_{1}>0$, there exists a flat totally geodesic rectangle $P_{1}$ with two of the edges $\left[p \gamma\left(t_{1}\right)\right]$ and $[p r]$. Hence there is a corresponding point $r_{1} \in$ $\partial \Omega_{f \circ \gamma\left(t_{1}\right)}$ such that $\left|r r_{1}\right|=\left|r \partial \Omega_{f \circ \gamma\left(t_{1}\right)}\right|$. By applying Theorem 0.5, we get another flat totally geodesic rectangle $P_{2}$ with two of the edges $\left[\gamma\left(t_{1}\right) \gamma\left(t_{2}\right)\right]$ and $\left[\gamma\left(t_{1}\right) r_{1}\right]$, for which there is a corresponding point $r_{2} \in \partial \Omega_{f \circ \gamma\left(t_{2}\right)}$, such that $\left|r_{1} r_{2}\right|=\left|r_{1} \partial \Omega_{f \circ \gamma\left(t_{2}\right)}\right|$. Next we will show that $P_{1} \cup P_{2}$ is a flat totally geodesic rectangle. There is a canonical map, $g: R=\left\{(x, y) \in \mathbb{R}^{2}|0 \leq x \leq| p r \mid, 0 \leq y \leq t_{2}\right\} \rightarrow P_{1} \cup P_{2}$, with $g((0,0))=p$, and $g\left(\left\{x=0,0 \leq y \leq t_{2}\right\}\right)=\gamma$. In order to show that $g$ is an isometry, it suffices to show that $\left|g\left(z_{1}\right) g\left(z_{2}\right)\right|=\left|z_{1} z_{2}\right|$, for any $z_{1}, z_{2} \in \partial R$. First one can easily see that $g\left(\left\{x, 0 \leq y \leq t_{2}\right\}\right)$ are all minimal geodesics; i.e., vertical directions are all isometry. The left cases are similar. We just show the case of $z_{1}=\left(0, t_{2}\right)$ and $z_{2}=(|p r|, 0)$. For $\gamma(t), 0 \leq t \leq t_{2}$ and $[p r]$, we can apply Theorem 0.5 to get another flat totally geodesic rectangle. Thus we get that $\left|\gamma\left(t_{2}\right) r\right|=\sqrt{t_{2}^{2}+|p r|^{2}}$; then $\left|g\left(z_{1}\right) g\left(z_{2}\right)\right|=\left|z_{1} z_{2}\right|$ follows.

Let $P=\bigcup_{i>0} P_{i}$. It follows that $P$ is a flat totally geodesic strip and there is a corresponding geodesic ray from $r$ with the desired property.

Note that the flat totally geodesic strip in Lemma (1.1.2) may not be unique; see the example in [42, 14.8].

Remark 1.3. Inspecting the proof of Lemma 1.2, one can see that when $\partial C \neq \emptyset$ the following holds: Let $x \in C$ be a point such that $|v C|=|v x|$. If $x \notin \partial C$, then $|v \xi|=\frac{\pi}{2}$, for any $\xi \in C$. We will use this observation in Section 4 .

\subsection{A reduction.}

The goal here is to reduce the proof of Theorem A to the simply connected case.

Let $A \in \operatorname{Alex}^{n}(\kappa)$. Recall that $p \in A$ is topologically regular if $\Sigma_{p} A$ is homeomorphic to a sphere. A topologically regular point has a neighborhood homeomorphic to a Euclidean ball. $A$ is called topologically regular if all points are topologically regular, and thus $A$ is a topological manifold. 
A point $p \in A$ is called topologically nice if the iterated spaces of directions, i.e., $\Sigma_{p} A, \Sigma_{v_{1}} \Sigma_{p} A, \ldots$, are all homeomorphic to spheres. $A$ is called topologically nice if all points are topologically nice. Topologically nice implies topologically regular, but the converse may not be true.

Example $1.4([21])$. Let $\Sigma^{3}$ be the Poincaré homology 3-sphere with constant curvature 1. Then the three-fold spherical suspension of $\Sigma^{3}, S^{3}\left(\Sigma^{3}\right) \in \operatorname{Alex}^{6}(1)$, is topologically regular but not topologically nice.

Lemma 1.5. If Theorem A holds for simply connected Alexandrov spaces, then it holds for any nonsimply connected Alexandrov spaces.

Proof. Let $\tilde{S}$ be the universal cover of $S$. Denote the covering map by $\phi$. Let $\phi^{*}(A)=\{(p, e) \in \tilde{S} \times A \mid \phi(p)=\pi(e)\} \subset \tilde{S} \times A$, with the induced topology. Then by a standard argument, we obtain that $\phi^{*}(A)$ is the universal cover of $A$, and $\tilde{\phi}: \phi^{*}(A) \rightarrow A$, defined by $\tilde{\phi}((p, e))=e$, is the covering map.

Endow $\phi^{*}(A)$ with the length metric (the lift metric of $A$; cf. [2, 3.4.2]) such that $\tilde{\phi}: \phi^{*}(A) \rightarrow A$ is a local isometry. Denote $\phi^{*}(A)$ by $\tilde{A}$. Then $\tilde{\pi}: \tilde{A} \rightarrow \tilde{S}$, with $\tilde{\pi}((p, e))=p$, is locally 1-Lipschitz.

First we assume that $C_{0}=S$.

Sublemma 1.6. Let $\tilde{\Omega}_{c}=\left\{(p, e) \in \tilde{S} \times \Omega_{c} \mid \phi(p)=\pi(e)\right\}$. Then $\tilde{\Omega}_{c} \subset \tilde{A}$ is convex.

Proof. For $x, y \in \tilde{\Omega}_{c}$, if $[x y] \varsubsetneqq \tilde{\Omega}_{c}$, by the construction of $\tilde{A}$, there exists a curve in $\tilde{\Omega}_{c}$ with length $\leq|x y|$, a contradiction. Thus we get the sublemma.

Let $\tilde{f}=\operatorname{dist}_{\partial \tilde{\Omega}_{c}}$. By the property of covering space, $\tilde{f}((p, e))=\left|(p, e), \partial \tilde{\Omega}_{c}\right|=$ $\left|e, \partial \Omega_{c}\right|=f(e)-c$. It follows that $\partial \tilde{\Omega}_{c}$ are level sets of $\tilde{f}$, and by the local isometry, $\left|\nabla \tilde{f}_{(p, e)}\right|=\left|\nabla f_{e}\right|$. Hence $\alpha(t)$ is an $f$-gradient curve if and only if $(p, \alpha(t))$ is an $\tilde{f}$-gradient curve.

If $\tilde{S}$ is compact, by the assumption of Lemma 1.5, we can see that $\tilde{\pi}$ is a submetry (since $\tilde{A}$ is topologically nice). Hence $\pi$ is also a submetry.

If $\tilde{S}$ is not compact, by the splitting theorem [23, there is an isometric splitting $\tilde{S}=\mathbb{R}^{k} \times S_{0}$, where $S_{0}$ is simply connected and compact, exactly as in the proof of the Riemannian case. It follows that $\tilde{A}=\mathbb{R}^{k} \times A_{0}$ and $\partial \tilde{\Omega}_{c}=\mathbb{R}^{k} \times \partial \Omega_{c}^{\prime}$. We claim that $\nabla_{\left(x, x_{0}\right)} \operatorname{dist}_{\partial \tilde{\Omega}_{c}} \in T_{x_{0}} A_{0}$, for any $\left(x, x_{0}\right) \in \tilde{A}$. Hence $\tilde{\pi}=\left(i d, \pi_{0}\right)$.

Since $\tilde{A}=\mathbb{R}^{k} \times A_{0}$ is topologically nice, we have that $A_{0}$ is topologically nice (see Remark 1.7), as can be seen in the proof of [32, Theorem D]. Thus by the assumption of Lemma 1.5, we know that $\pi_{0}: A_{0} \rightarrow S_{0}$ is a submetry. It follows that $\tilde{\pi}$ is a submetry.

Finally, we will verify the claim: for any $x_{0} \in A_{0}$ and $y=\left(y_{1}, x_{0}\right) \in \mathbb{R}^{k} \times A_{0}$, we have that $\Sigma_{y} \tilde{A}=S^{k}(\Sigma)$, where $\Sigma=\Sigma_{x_{0}} A_{0}$. Let $v$ be a point such that $\left|v \Uparrow_{y}^{\partial \tilde{\Omega}_{c}}\right|=\max _{w \in \Sigma_{y} \tilde{A}}\left\{\left|\Uparrow_{y}^{\partial \tilde{\Omega}_{c}} w\right|\right\}$. Since $\partial \tilde{\Omega}_{\bar{c}}=\mathbb{R}^{k} \times \partial \Omega_{\bar{c}}^{\prime}$, for any $\bar{c}<a_{0}$, we have that

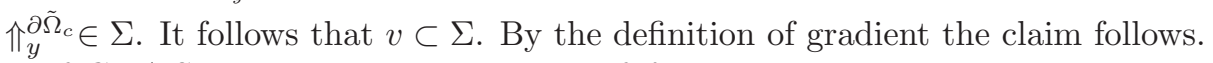

If $C_{0} \neq S$, consider $\operatorname{dist}_{\partial \Omega_{a_{0}}}$ instead of $f$; we can get the same conclusion.

Remark 1.7. If $A$ is only topologically regular, then $A_{0}$ may not be a topological manifold, even if $\tilde{A}=\mathbb{R}^{k} \times A_{0}$ is a topological manifold ([21]). 


\section{Proof of Theorem A}

In our proof of Theorem A, the following structural results on spaces of directions of points on soul plays a crucial role.

Proposition 2.1. Let the assumptions be as in Theorem A. For $p \in S$, let $\Sigma_{0}^{p}=$ $\Sigma_{p} S$, and let $\Sigma_{1}^{p}=\left\{v \in \Sigma_{p} A|| v \Sigma_{p} S \mid \geq \frac{\pi}{2}\right\}$. Then

(2.1.1) $\Sigma_{0}^{p}$ is homeomorphic to a sphere.

(2.1.2) $\Sigma_{1}^{p}$ is conver 1 and isometric to one of the following: $S^{1}(r)$ with $r \leq 1,[a b],\{v\},\left\{v_{1}, v_{2}\right\}$ with $\left|v_{1} v_{2}\right|=\pi$.

(2.1.3) With the restricted metric, $\left[\Sigma_{0}^{p} \Sigma_{1}^{p}\right]=\Sigma_{0}^{p} * \Sigma_{1}^{p}$ with the standard join metric 2

(2.1.4) When $\Sigma_{1}^{p}=[a b]$, if there exists a subset $E$ of $\Sigma_{1}^{p}$ such that $B\left(E, \frac{\pi}{2}\right)=\Sigma_{p} A$, then $a, b \in E$. If $|a b|=\pi$ with o the middle point of $[a b]$, then for any $x \in \Sigma_{p} A$ such that $\mid$ ox $\mid \leq \frac{\pi}{2}, x \in\left[\Sigma_{0}^{p} \Sigma_{1}^{p}\right]$.

Because the proof of Proposition 2.1 is technical and long, we will postpone the proof to the next section. Below we shall prove Theorem A by assuming Proposition 2.1 .

\subsection{Proof of Key Lemma 0.7.}

Recall that

$F_{v}=\bigcup\{x \mid x \in$ flat totally geodesic strips in $A$ spanned by $\sigma$ and all minimal geodesics in $S$ from $p$ to all the points in $S$ \},

where $p \in S, v \in \Uparrow_{p}^{\partial \Omega_{c}} \subset \Sigma_{1}^{p}(c<\max f), \sigma(t) \subset A$ is a ray with $\sigma(0)=p$ and $\sigma^{+}(0)=v($ see Lemma (1.1.1)), and if $\gamma \subset S$ is a minimal geodesic from $p$ to $q \in S$, then $\{\sigma, \gamma\}$ determines a unique flat totally geodesic strip (see Lemma (1.1.2)). The uniqueness follows from Proposition (2.1.3); otherwise, it will violate the join of $\left[\Sigma_{0}^{p} \Sigma_{1}^{p}\right]$.

Let $\alpha$ be the other ray from $q$ which bounds the flat totally geodesic strip, with $\bar{v}=\alpha^{+}(0) \in \Uparrow_{q}^{\partial \Omega_{c}}$. We then define a map $\phi_{[p q]}: \Uparrow_{p}^{\partial \Omega_{c}} \rightarrow \Uparrow_{q}^{\partial \Omega_{c}}$ by $\phi_{[p q]}(v)=\bar{v}$. Note that $\phi_{[p q]}$ may depend on the choice of $[p q]$.

Observe that for all $q \in S, \phi_{[p q]}$ is independent of $[p q]$ if and only if $F_{v}$ is isometric to $S \times \mathbb{R}_{+}^{1}$; i.e., Key Lemma 0.7 holds.

To prove the independency, we will first show that $\phi_{[p q]}$ is an isometry. (We point out that the method we use in the proof of Lemma 2.2 below was previously used in [29].)

Lemma 2.2. Let the assumptions be as in Theorem A. For every $x \in S$ and every $y \in S, \phi_{[x y]}: \Uparrow_{x}^{\partial \Omega_{c}} \rightarrow \Uparrow_{y}^{\partial \Omega_{c}}$ is an isometry for any minimal geodesic [xy].

Proof. For $u, v \in \uparrow_{x}^{\partial \Omega_{c}}$ and $\varepsilon>0$, let $\bar{v}_{\varepsilon}=\left|y, \exp _{x}(\varepsilon v)\right| \uparrow_{y}^{\exp _{x}(\varepsilon v)}$, where $\exp _{x}$ : $T_{x} \rightarrow A$ is the usual exponential map, and $\uparrow_{y}^{\exp _{x}(\varepsilon v)}$ is the direction of the minimal geodesic contained in the flat totally geodesic strip determined by $\exp _{x}(t v), t \geq 0$, and $[x y]$. Similarly for $\bar{u}_{\varepsilon}$, with $\varepsilon u$ instead of $\varepsilon v$ in the definition of $\bar{v}_{\varepsilon}$.

Since by Proposition (2.1.3) and by the property of flat totally geodesic strips, we have that $\left|\bar{v}_{\varepsilon}^{\perp} \bar{u}_{\varepsilon}^{\perp}\right|_{T_{y}}=\left|\bar{v}_{\varepsilon} \bar{u}_{\varepsilon}\right|_{T_{y}} \geq\left|\exp _{x}(\varepsilon v) \exp _{x}(\varepsilon u)\right|=\varepsilon|u v|_{T_{x}}+o(\varepsilon)$, where $\perp$ is the orthogonal projection to $C\left(\Sigma_{1}^{y}\right)$ (Euclidean cone over $\Sigma_{1}^{y}$ ).

\footnotetext{
${ }^{1}$ See Remark (4.3.2) and (4.3.1).

${ }^{2}$ See Definition 4.1 .
} 
Let $\bar{u}=\lim _{\varepsilon \rightarrow 0} \frac{1}{\varepsilon} \bar{u}_{\varepsilon}^{\perp}=\phi_{[x y]}(u)$ and $\bar{v}=\lim _{\varepsilon \rightarrow 0} \frac{1}{\varepsilon} \bar{v}_{\varepsilon}^{\perp}=\phi_{[x y]}(v)$. Then $|\bar{u} \bar{v}|_{T_{y}} \geq|u v|_{T_{x}}$, and $|\bar{u}|=|u|,|\bar{v}|=|v|$. Thus we get that $\left|\phi_{[x y]}(u) \phi_{[x y]}(v)\right|_{\Sigma_{y}} \geq|u v|_{\Sigma_{x}}$.

Similarly, the opposite inequality holds. Hence $\left|\phi_{[x y]}(u) \phi_{[x y]}(v)\right|_{\Sigma_{y}}=|u v|_{\Sigma_{x}}$.

Remark 2.3. It seems that Lemma 2.2 can be strengthened so that the isometric class of $\Sigma_{1}^{p}$ is independent of $p$.

Proof of Key Lemma 0.7. Define a map $\psi: F_{v} \rightarrow S \times \mathbb{R}_{+}^{1}, \psi\left(\exp _{q}\left(t \phi_{[p q]}(v)\right)\right)=$ $(q, t)$. As pointed out earlier, $\psi$ is an isometry if $\phi_{[p q]}(v)$ is independent of $q \in S$. In view of the simple connectedness of $S$ (because $A$ is simply connected), first we will show that $F_{v}$ is a product locally, so it suffices to show that locally $\phi_{[p q]}(v)$ is independent of $q \in S$ (all $p$ ). Precisely, for $x \in S$, there exists $\varepsilon>0$ ( $\varepsilon$ depends on $x)$ such that for any $y, z \in B(x, \varepsilon), g=\phi_{[z x]} \circ \phi_{[y z]} \circ \phi_{[x y]}=i d$.

If $\Sigma_{1}^{x}=\{v\}$, then $\Uparrow_{x}^{\partial \Omega_{c}}=\Sigma_{1}^{x}$. Thus each point in $S$ has just one special normal ray to $S$, so clearly $g=i d$.

For other cases we will argue by contradiction. Suppose that for a sequence $\varepsilon_{i} \rightarrow 0$, there exist $y_{i}, z_{i} \in B\left(x, \varepsilon_{i}\right), g_{i}=\phi_{\left[z_{i} x\right]} \circ \phi_{\left[y_{i} z_{i}\right]} \circ \phi_{\left[x y_{i}\right]} \neq i d$.

If $\Sigma_{1}^{x}=\left\{v_{1}, v_{2}\right\}$ with $\left|v_{1} v_{2}\right|=\pi$, then $g_{i}\left(v_{1}\right)=v_{2}$, and by the property of flat totally geodesic strips, we have that $\left|\exp _{x}\left(v_{1}\right) \exp _{x}\left(g_{i}\left(v_{1}\right)\right)\right| \leq(|x y|+|y z|+|z x|) \leq$ $4 \varepsilon_{i}$. When $\varepsilon_{i} \rightarrow 0$, we get a contradiction, since $\left|\exp _{x}\left(v_{1}\right) \exp _{x}\left(v_{2}\right)\right|>0$, or geodesic branches.

If $\Sigma_{1}^{x}=S^{1}(r)$ with $r \leq 1$, by Lemma 2.4 below, we have that every $g_{i}$ is the restriction of an isometry, $\bar{g}_{i}: S^{1} \rightarrow S^{1}$, which is a rotation or a reflection. By passing to a subsequence, we can suppose that every $\bar{g}_{i}$ is a rotation or every $\bar{g}_{i}$ is a reflection.

(a): Every $\bar{g}_{i}$ is a rotation.

For $v \in \Uparrow_{x}^{\partial \Omega_{c}},\left|\exp _{x}(t v) \exp _{x}\left(g_{i}(t v)\right)\right| \rightarrow 0$, for any $t \geq 0$, as $\varepsilon_{i} \rightarrow 0$, which can be seen in the above case. Hence $\left|v g_{i}(v)\right| \rightarrow 0$, i.e. $\left|v \bar{g}_{i}(v)\right| \rightarrow 0$, as $\varepsilon_{i} \rightarrow 0$. Then by the closeness of $\Uparrow_{x}^{\partial \Omega_{c}}$, we can get that $\uparrow_{x}^{\partial \Omega_{c}}=S^{1}$. This is a contradiction, since $\mathscr{F} \neq A$ and Lemma 2.2 imply that $\Uparrow_{q}^{\partial \Omega_{c}} \neq S^{1}$, for any $q \in S$.

(b): Every $\bar{g}_{i}$ is a reflection.

By passing to a subsequence, we can assume that $\bar{g}_{i} \rightarrow h$, which is also a reflection. Observe that there is $v \in \uparrow_{x}^{\partial \Omega_{c}}$ such that $v \neq h(v)$ or $g_{i}$ will be equal to $i d$, a contradiction. Similarly, we have that $\left|\exp _{x}(t v) \exp _{x}\left(g_{i}(t v)\right)\right| \rightarrow 0$, for any $t \geq 0$, as $\varepsilon_{i} \rightarrow 0$. Hence $\left|v g_{i}(v)\right| \rightarrow 0$, i.e. $\left|v \bar{g}_{i}(v)\right| \rightarrow 0$, as $\varepsilon_{i} \rightarrow 0$. Thus we have that $v=h(v)$, a contradiction.

If $\Sigma_{1}^{x}=[a b]$, likewise by Lemma 2.4, each $g_{i}$ can be the restriction of the reflection of $[a b]$. Similarly as above, we can get the conclusion.

Then $\left.F_{v}\right|_{B(x, \varepsilon)}$ is a product. (We call $B(x, \varepsilon)$ a local product neighborhood of $x$.)

Finally, we will show that $F_{v}$ is a product globally. For any $q, r \in S$ and for three fixed geodesics $[x q],[q r]$ and $[r x]$, let $\gamma=[x q] \cup[q r] \cup[r x]$. Since $\pi_{1}(S)=0, \gamma$ is homotopic to a point. Let $H:[0,1] \times[0,1] \rightarrow S$ be a homotopy, with $H(t, 1)=$ $\gamma(t), H(t, 0)=x, H(0, s)=x$ and $H(1, s)=x$. Let $\left\{s_{0}=0<s_{1}<\cdots<s_{n}=1\right\}$ and $\left\{t_{0}=0<t_{1}<\cdots<t_{n}=1\right\}$ be two partitions of $[0,1]$ such that $H\left(\left[s_{i}, s_{i+1}\right] \times\right.$ $\left.\left[t_{j}, t_{j+1}\right]\right) \subset U_{z}$, a closed convex neighborhood of some $z \in S$ which is contained in the local product neighborhood of $z$. Let $\sigma_{i}=\bigcup_{j=0}^{n-1}\left[H\left(s_{i}, t_{j}\right) H\left(s_{i}, t_{j+1}\right)\right]$. There are corresponding $g_{i}$. We can see that $g_{i}(w)$, for any $w \in \Uparrow_{x}^{\partial \Omega_{c}}$, are the same for 
any $i$. It follows that $g_{1}(w)=w$, i.e., $g_{1}=i d$. Hence $\phi_{[r x]} \circ \phi_{[q r]} \circ \phi_{[x q]}=i d$. Thus we can get that $\psi$ is an isometric map from $S \times \mathbb{R}_{+}^{1}$ to $F_{v}$.

Lemma 2.4. Let $M \stackrel{\text { homeo }}{\cong} S^{1}$ or an interval and with intrinsic metric, let $N \subset M$ be a subset and let $g: N \rightarrow N$ be an isometry, where $N$ is with the restricted metric. Then $g$ can be extended to an isometry $\bar{g}: M \rightarrow M$.

Proof. We show only the case of $M \stackrel{\text { homeo }}{\cong} S^{1}$, similarly for an interval.

If there exist $v, w \in N$ such that $v, w$ are not antipodal, then for any $u \in M, u$ is uniquely determined by $|u v|,|u w|$. Thus $g$ is uniquely determined by $g(v), g(w)$. Hence $g$ can be extended to $\bar{g}$, by $\bar{g}(u)=x$, where $x$ is the unique point such that $|x g(v)|=|u v|$ and $|x g(w)|=|u w|$.

If not, then $N=\{v, w\}$ with $v, w$ antipodal. Clearly $g$ is extendable.

Next we will show

Lemma 2.5. For every $x \in F_{u}$ and every $y \in F_{u},[x y] \subset F_{u}$.

In the proof of Lemma 2.5, we need the following lemma.

Lemma 2.6 ([14, 2.4(ii')]). Let $X \in \operatorname{Alex}^{m}(\kappa)$. For two minimal geodesics $[x z]$ and $[x y]$, if $\angle(\uparrow \underset{x}{z}, \uparrow \underset{x}{y})=\tilde{L}(z, x, y)$, then there is a $[z y]$ such that $[x z],[x y],[z y]$ bound a totally geodesic surface which is isometric to a geodesic triangle in $S^{2}(\kappa)$.

Proof of Lemma 2.5. Set $\pi(x)=\bar{x}$ and $\pi(y)=\bar{y}$. If $x, y \in S$, by the construction of $S$, we have that $[x y] \subset S$.

For other cases we will argue by contradiction. Suppose that there exist $x, y \in F$ such that there exists $[x y]$ which doesn't belong to $F$. Then $[x y]^{\circ} \cap F=\emptyset$, where $[x y]^{\circ}$ denotes $[x y]-\{x, y\}$, or geodesic will branch.

If $x \in S, y \in(S, a)$, with $a \neq 0$, let $r \in F_{u}, r \neq y$, be a point such that $\pi(r)=\bar{y}$ and $|r \pi(r)|>|y \bar{y}|$. Then $\pi=L\left(\uparrow_{y}^{x}, \uparrow_{y}^{\bar{y}}\right)+\angle\left(\uparrow_{y}^{x}, \uparrow_{y}^{r}\right) \geq \tilde{L}(x, y, \bar{y})+\tilde{L}(x, y, r)=\pi$, where the last equality is from the construction of $F$. Hence $\angle\left(\uparrow_{y}^{x}, \uparrow \frac{\bar{y}}{y}\right)=\tilde{L}(x, y, \bar{y})$. It follows from Lemma 2.6 that $\{x, y, \bar{y}\}$ bounds another flat totally geodesic triangle, which contradicts the structure of $\Sigma_{\bar{y}} A$ (Proposition (2.1.3)), since $[x \bar{y}] \subset S$.

If $x, y \in(S, a)$, with $a \neq 0$, then similarly as above we have that $\angle\left(\uparrow \begin{array}{c}y \\ x\end{array}, \uparrow_{x}^{\bar{x}}\right)=$ $\tilde{L}(y, x, \bar{x})=\frac{\pi}{2}$. Therefore there exists a flat triangle bounded by $y, x, \bar{x}$ for the given $[x y]$, with $[y \bar{x}]^{\circ} \nsubseteq F$. By the above case, we get a contradiction.

If $x \in(S, a), y \in(S, b)$, with $a \neq 0, b \neq 0$ and $a \neq b$, without loss of generality, we can assume that $a<b$. Let $s=[y \bar{y}] \cap(S, a)$. Similarly as the above two cases, we can also get a contradiction.

As seen following Lemma 1.2, the remaining case in the proof of Theorem A is that $\mathscr{F} \neq A$ and $\pi_{1}(A)=0$, which implies that $\Uparrow_{p}^{\partial \Omega_{c}} \neq S^{1}$.

\subsection{The concavity of $\operatorname{dist}_{F}$.}

As seen in the introduction, $F$ is the union of several $F_{v}$ 's. We point out that the selection of these $F_{v}$ 's is crucial for the desired concavity of $\operatorname{dist}_{F}$; see the following for details.

For $p \in S$, by the first variation formula for the Busemann function, $d_{p} f(v)=$ $-\left\langle\Uparrow_{p}^{\partial \Omega_{c}}, v\right\rangle \leq 0, v \in \Sigma_{p}$, we see that $\Uparrow_{p}^{\partial \Omega_{c}}$ is $\frac{\pi}{2}$-dense in $\Sigma_{p}$ and thus $\frac{\pi}{2}$-dense in $\Sigma_{1}^{p}$. 
Lemma 2.7. There is $N^{\prime}=\left\{v_{i}\right\}_{1 \leq i \leq l} \subset \Uparrow_{p}^{\partial \Omega_{c}}, l \leq 3$, such that $N^{\prime}$ is $\frac{\pi}{2}$-dense in $\Sigma_{1}^{p}$, and $\phi_{[p q]}\left(N^{\prime}\right)$ is also $\frac{\pi}{2}$-dense in $\Sigma_{1}^{q}$, for any $q \in S$.

Proof. First for the selection of $N^{\prime}$ : if $\Sigma_{1}^{p}=\{v\}$, then $\Uparrow_{p}^{\partial \Omega_{c}}=\Sigma_{1}^{p}$. Let $N^{\prime}=\Uparrow_{p}^{\partial \Omega_{c}}$.

If $\Sigma_{1}^{p}=\left\{v_{1}, v_{2}\right\}$ with $\left|v_{1} v_{2}\right|=\pi$, then $v_{1}, v_{2} \in \Uparrow_{p}^{\partial \Omega_{c}}$. Indeed, since $\Uparrow_{p}^{\partial \Omega_{c}} \neq \emptyset$, one of them, say $v_{1}$, must be in $\Uparrow_{p}^{\partial \Omega_{c}}$. Suppose that $v_{2} \notin \Uparrow_{p}^{\partial \Omega_{c}}$; then $d_{p} f\left(v_{2}\right)=1>0$, a contradiction. Let $N^{\prime}=\Uparrow_{p}^{\partial \Omega_{c}}$.

If $\Sigma_{1}^{p}=[a b]$, since $B\left(\Uparrow_{p}^{\partial \Omega_{c}}, \frac{\pi}{2}\right)=\Sigma_{p} A$, by Proposition (2.1.4), we have that $a, b \in \Uparrow_{p}^{\partial \Omega_{c}}$. Let $N^{\prime}=\{a, b\}$. Then $N^{\prime}$ is obviously a $\frac{\pi}{2}$-dense subset of $\Sigma_{1}^{p}$.

If $\Sigma_{1}^{p}=S^{1}(r)$ with $r \leq 1$, we choose $v \in \Uparrow_{p}^{\partial \Omega_{c}}$ arbitrarily, and consider the antipodal point of $v$, denoted by $w$. If $w \in \Uparrow_{p}^{\partial \Omega_{c}}$, let $N^{\prime}=\{v, w\}$. If $w \notin \Uparrow_{p}^{\partial \Omega_{c}}$, let $v_{1}, v_{2} \in \Uparrow_{p}^{\partial \Omega_{c}}$ be the farthest points to $w$ from both sides respectively in $\Sigma_{1}^{p}$. It follows that $v, v_{1}, v_{2}\left(v\right.$ may be equal to $v_{1}$ or $\left.v_{2}\right)$ form a $\frac{\pi}{2}$-dense subset of $\Sigma_{1}^{p}$. Let $N^{\prime}=\left\{v, v_{1}, v_{2}\right\}$.

For the second part of the lemma: If $\Sigma_{1}^{p}=\{v\}$ or $\left\{v_{1}, v_{2}\right\}$, by Lemma 2.2 one can deduce that $N^{\prime}$ has the desired property.

If $\Sigma_{1}^{p}=[a b]$, suppose that there is $q \in S$ such that $\phi_{[p q]}\left(N^{\prime}\right)$ is not a $\frac{\pi}{2}$-dense subset of $\Sigma_{1}^{q}$. Then there is $w \in \Uparrow_{p}^{\partial \Omega_{c}}$ such that $\left|\phi_{[p q]}(w) \phi_{[p q]}(a)\right|+\left|\phi_{[p q]}(w) \phi_{[p q]}(b)\right|>$ $|a b|$, a contradiction, since by Lemma $2.2, \phi_{[p q]}$ is an isometry when restricted to $\Uparrow_{p}^{\partial \Omega_{c}}$.

If $\Sigma_{1}^{p}=S^{1}(r)$ with $r \leq 1$, then from the choosing method, we have that for any $u \in \Uparrow_{p}^{\partial \Omega_{c}}$, either $|u v|+\left|u v_{1}\right|=\left|v v_{1}\right|$ or $|u v|+\left|u v_{2}\right|=\left|v v_{2}\right|$. Suppose that there is $q \in S$ such that $\phi_{[p q]}\left(N^{\prime}\right)$ is not a $\frac{\pi}{2}$-dense subset of $\Sigma_{1}^{q}$. Then there is $w \in \Uparrow_{p}^{\partial \Omega_{c}}$ such that $\left|\phi_{[p q]}(w) \phi_{[p q]}(v)\right|+\left|\phi_{[p q]}(w) \phi_{[p q]}\left(v_{1}\right)\right|>\left|v v_{1}\right|$ and $\left|\phi_{[p q]}(w) \phi_{[p q]}(v)\right|+\left|\phi_{[p q]}(w) \phi_{[p q]}\left(v_{2}\right)\right|$ $>\left|v v_{2}\right|$, a contradiction.

Thus we finish the proof of the lemma.

Lemma 2.8. Let $F=\bigcup_{v \in N^{\prime}} F_{v}$. The distance function, $\operatorname{dist}_{F}$, is concave in $D=X \backslash F$.

Remark 2.9. Observe that if the boundary points of each component $D_{i}$ are "true" boundary points, i.e., are not interior points in the closure $\bar{D}_{i}$, and $\bar{D}_{i}$ is convex, then it follows that $\operatorname{dist}_{F}$ is concave in $D$. In our case, we show that even if a component of $D$ may not be convex, $\operatorname{dist}_{F}$ is still concave. For example, let $T=\left\{(x, y) \in \mathbb{R}^{2} \mid x \geq 0, y \geq 10 x\right\} \cup\left\{(x, y) \mid x \leq 0, y \geq x^{2}\right\}$, the metric product, and $A=\operatorname{Doub}(T) \times S^{2}(1) \in \operatorname{Alex}^{4}(0)$. Then $F=\operatorname{Doub}(\{x=0, y \geq 0\})$ serves as an example.

In the proof of Lemma 2.8, we need the following lemma, which is an analogue to the totally geodesic property in Riemannian geometry. (Lemma 2.10 below is from a helpful discussion with Shicheng $\mathrm{Xu}$.)

Lemma 2.10. Let $X \in \operatorname{Alex}^{n}(\kappa)$, and let $Y$ be a closed subset of $X$ such that for $x, y \in Y$, any $[x y] \subset Y$. Then for any $p \in Y$ and $q \in Y \backslash \partial Y$, we have that $\nabla_{q} \operatorname{dist}_{p} \in T_{q} Y$.

We don't know whether Lemma 2.10 is true for a convex subset or not.

Proof of Lemma 2.10. If $\nabla_{q}$ dist $_{p}=0$, nothing needs to be proved. Hence we can assume $\nabla_{q}$ dist $_{p} \neq 0$. Since $\frac{\nabla_{q} \text { dist }_{p}}{\mid \nabla_{q} \text { dist }_{p} \mid}=\left\{u \in \Sigma_{q} X|| u \Uparrow_{q}^{p} \mid=\max _{v \in \Sigma_{q}}\left\{\left|v \Uparrow_{q}^{p}\right|\right\}\right.$, and by 
the condition of the lemma, $\Uparrow_{q}^{p} \subset \Sigma_{q} Y$, it suffices to show that for $V \subset \Sigma_{q} Y$, we can let $w \in \Sigma_{q} X$ be a point such that $|V w|=\max \{|V|\}>,\frac{\pi}{2}$. Then we have that $w \in \Sigma_{q} Y$.

Argue by contradiction. Suppose that $w \notin \Sigma_{q} Y$. Choose $w_{0} \in \Sigma_{q} Y$ such that $\left|w w_{0}\right|=\left|w \Sigma_{q} Y\right|$. By Lemma 1.2, $\left|\uparrow w_{w_{0}} \bar{v}\right|=\frac{\pi}{2}$, for any $\bar{v} \in \Sigma_{w_{0}} \Sigma_{q} Y$ and $\left|w w_{0}\right| \leq \frac{\pi}{2}$. Hence $\tilde{L}\left(\tilde{w}, \tilde{w}_{0}, \tilde{V}\right) \leq \frac{\pi}{2}$. By hinge comparison, we have that $\left|V w_{0}\right|>\frac{\pi}{2}$. It follows that $|V w|<\left|V w_{0}\right|$, a contradiction to the choice of $w$.

Recall a standard fact in topology (cf. [5]): If $X \subset S^{m}$ is a closed $(m-1)$ topological manifold as a subspace, then $S^{m}-X$ has two connected components, each having $X$ as its set boundary. We say that $X$ separates $S^{m}$.

Proof of Lemma 2.8. Given $q \in D$, let $\gamma(t) \subset D$ be a minimal geodesic with $\gamma(0)=$ $q$, and let $x \in F$ be a point such that $|q x|=|q F|$. By a standard contradiction argument, one can get that for $v=\uparrow_{x}^{\gamma(t)} \in \Sigma_{x} A$, when $t$ is small enough, there is $\uparrow_{x}^{q}$ such that $\left|v \uparrow_{x}^{q}\right|<\frac{\pi}{2}$. (Note that for different $t, \uparrow_{x}^{q}$ may be different.)

From the proof of the concavity of distance function to the boundary of an Alexandrov space (cf. [30, Theorem 3.3.1], [7, Lemma 3.1]), one can deduce that if $F$ satisfies the following two conditions:

(i) there is $\bar{w} \in \Sigma_{x} F$ such that $\left|\uparrow_{x}^{q} \bar{w}\right|=\left|\uparrow_{x}^{q} v\right|+|v \bar{w}|=\frac{\pi}{2}$,

(ii) there is a radial curve, $\sigma:[0, \varepsilon] \rightarrow F$, with $\sigma(0)=x$ and $\sigma^{+}(0)=\bar{w}$, for some $\varepsilon>0$,

then $\operatorname{dist}_{F}$ is concave in $D$.

Thus it suffices to check that $F$ satisfies the two conditions.

For condition (i): if $x \in S$, by the first variation formula, we have that $\left|\uparrow_{x}^{q} \Sigma_{x} F\right| \geq \frac{\pi}{2}$. Particularly, $\left|\uparrow_{x}^{q} \Sigma_{x} S\right| \geq \frac{\pi}{2}$; thus $\uparrow_{x}^{q} \in \Sigma_{1}^{x}$. Hence $\Sigma_{1}^{x}=S^{1}$ or $[a b]$ with $|a b|=\pi$ and $\uparrow_{x}^{q}$ is the middle point of $[a b]$. By Lemma 2.7, we have that for any $v \in \Sigma_{x},\left|v \phi_{[p x]}\left(N^{\prime}\right)\right| \leq \frac{\pi}{2}$. Hence there are two of $N^{\prime}$, say $v_{1}, v_{2}\left(v_{1}\right.$ may be equal to $\left.v_{2}\right)$, such that $\left|\phi_{[p x]}\left(v_{1}\right) \uparrow_{x}^{q}\right|=\frac{\pi}{2},\left|\phi_{[p x]}\left(v_{2}\right) \uparrow_{x}^{q}\right|=\frac{\pi}{2}$. Since $\left|\uparrow_{x}^{q} v\right|<\frac{\pi}{2}$, by Proposition (2.1.4), we have that $v \in\left[\Sigma_{0}^{x} \Sigma_{1}^{x}\right]$. Thus by Proposition (2.1.3), we have that $v \in\left[w_{1} w_{0}\right]$, for some $w_{i} \in \Sigma_{i}^{x}$. We can suppose that $w_{1} \in\left[\uparrow_{x}^{q} \phi_{[p x]}\left(v_{2}\right)\right]$. Then $\triangle\left(\uparrow_{x}^{q}, w_{0}, \phi_{[p x]}\left(v_{2}\right)\right)$ is isometric to a triangle with three side lengths $\frac{\pi}{2}$ on $S^{2}(1)$. Hence there is $\bar{w} \in \Sigma_{x} F$ such that $\left|\uparrow_{x}^{q} \bar{w}\right|=\left|\uparrow_{x}^{q} v\right|+|v \bar{w}|=\frac{\pi}{2}$.

If $x \bar{\in} S, \Sigma_{x} F=S\left(\Sigma_{x} S\right) \stackrel{\text { homeo }}{\simeq} S^{n-2}$ is convex in $\Sigma_{x} A$ and separates $\Sigma_{x} A$, and by the first variation formula, $\left|\uparrow_{x}^{q} \Sigma_{x} F\right| \geq \frac{\pi}{2}$. Then by Lemma 4.19 below, we have that there is $\bar{w} \in \Sigma_{x} F$ such that $\left|\uparrow_{x}^{q} \bar{w}\right|=\left|\uparrow_{x}^{q} v\right|+|v \bar{w}|=\frac{\pi}{2}$.

For condition (ii): since $\bar{w} \in \Sigma_{x} F$, without loss of generality, we can assume that $\bar{w} \in \Sigma_{x} F_{v_{1}}$. Since $\left(\Sigma_{x} F_{v_{1}}\right)^{\prime}$ is dense in $\Sigma_{x} F_{v_{1}}$, there are $q_{i} \in F_{v_{1}}$ such that $q_{i} \rightarrow x$ and $\uparrow_{x}^{q_{i}} \rightarrow \bar{w}$. Let $\sigma_{i}$ be the radial curve at $q_{i}$ with respect to $x$. By [1, Chapter 15] or [27, 3.4], we know that if we can show that $\lim _{i \rightarrow \infty} \sigma_{i}([0, \varepsilon])=\sigma([0, \varepsilon]) \subset F_{v_{1}}$ for some small $\varepsilon>0$, then $\sigma$ is the desired radial curve.

If $x \bar{\in} S$, by Lemma 2.10, we can get the desired radial curve. If $x \in S$ and $\bar{w} \in \Sigma_{x} S$, similarly by Lemma 2.10 , we can get the desired radial curve in $S$. If $x \in S$ and $\bar{w} \notin \Sigma_{x} S$, we can choose $q_{i} \in F_{v_{1}}-S$. We claim that $\sigma_{i}([0, \infty)) \subset F_{v_{1}}$. Therefore we can get the desired radial curve. 
Finally, we will verify the claim by showing that $\sigma_{i}$ are farther and farther away from $\partial F_{v_{1}}$. The reason is that

$$
\left(\operatorname{dist}_{S} \circ \sigma_{i}(t)\right)^{+}=-\left\langle\uparrow_{\sigma_{i}(t)}^{\pi\left(\sigma_{i}(t)\right)}, \sigma_{i}^{+}(t)\right\rangle=-\left\langle\uparrow_{\sigma_{i}(t)}^{\pi\left(\sigma_{i}(t)\right)}, \frac{\left|x \sigma_{i}(t)\right|}{t} \nabla_{\sigma_{i}(t)} \operatorname{dist}_{x}\right\rangle \geq 0 ;
$$

the last inequality is because of the symmetry of $F$ locally. We have that $\uparrow_{q}^{x}$ are in the same half sphere as $\uparrow_{q}^{\pi(q)}=\uparrow_{q}^{\partial F_{v_{1}}}$ in $\Sigma_{q} F$, for any $q \in F$.

The lemma thus follows.

\subsection{Extending dist $_{F}$-gradient flows.}

Since $\operatorname{dist}_{F}$ is concave in $D=A \backslash F$, for each $x \in D$ there is a unique $\operatorname{dist}_{F^{-}}$ gradient curve from $x$. We call a gradient curve maximal if it is not a proper subset of another gradient curve. Note that any maximal gradient curve has empty intersection with $F$. We will extend maximal gradient curves to include points in $F$ so that each point in $F-(S, 0)$ is contained in two extended maximal gradient curves. This property plus the simple connectedness of $A$ allow us to choose one such curve for each point in $F-(S, 0)$ such that we can define a "flow", $\Psi_{a}^{t}:(S, a>$ $0) \rightarrow A$, by $\Psi_{a}^{t}((s, a))=\gamma_{a}(t)$, where $\gamma$ is the chosen extended maximal gradient curve at $(s, a)$, passing any given extended maximal gradient curve at any given $\left(s_{0}, a\right)$. Our goal is to show that $\Psi_{a}^{t}$ is 1-Lipschitz.

To carry out the above, the key is to establish the local separation property for $F-(S, 0)$ (see Lemma 2.11) and the local 1-Lipschitz property for $\Psi_{a}^{t}$.

Before moving on, we need the following two lemmas.

Lemma 2.11. For any $q \in(S, a) \subset F_{u}, a \neq 0, B_{F}(q, r)$ separates $B(q, r)$, for $r$ small enough, where $B_{F}(q, r)$ is a closed $r$-ball in $F$.

Proof. For $r$ small enough, we can assume that $B(q, r) \cap F=B(q, r) \cap F_{v_{1}}$. By the local version of Perelman's stability theorem ([24, 4.7]) and Proposition (2.1.1), we can choose $r$ sufficiently small such that $B(q, r)$ is homeomorphic to an $r$-ball on $T_{q} A$, which is homeomorphic to $D^{n}$, and $B_{F}(q, r)$ is homeomorphic to an $r$-ball on $T_{q} F$, which is homeomorphic to $D^{n-1}$. By definition, $B_{F}(q, r) \cap \operatorname{Fr}(B(q, r))=$ $\left(\operatorname{Fr}\left(B_{F}(q, r)\right)\right.$ in $\left.F\right)$, which is homeomorphic to $S^{n-2}$. By considering the double of $B(q, r)$, we get that $B_{F}(q, r)$ separates $B(q, r)$.

Let $q, r$ be as in Lemma 2.11, and let $\bar{U}_{q} \subset B(q, r)$ be a convex closed neighborhood of $q$. Then $B_{F}(q, r)$ separates $\bar{U}_{q}$ into two components $G_{q 1}, G_{q 2}$.

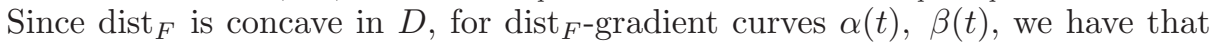
$|\alpha(t) \beta(t)|$ is 1-Lipschitz if there exists a minimal geodesic joining $\alpha(t)$ and $\beta(t)$, for any $t$, in the domain $D$. The following property will guarantee the local 1-Lipschitz property for $\Psi_{a}^{t}$.

Lemma 2.12. For $x, y$ in the interior of the same component, say $G_{q 1} \backslash \partial \bar{U}_{q}$ (denoted by $\left.G_{b 1}^{\circ}\right)$, we have that $[x y] \cap F=\emptyset$.

In the proof of Lemma 2.12, we need the following theorem about the relationship between the boundary of a convex subset as an Alexandrov space and the set boundary.

Theorem $2.13([1])$. Let $C \subset X \in \operatorname{Alex}^{m}(\kappa)$ be a convex closed subset $(C \in$ $\operatorname{Alex}^{m}(\kappa)$ with the induced metric). If $C$ has a nonempty interior, then $\partial C=$ $\operatorname{Fr}(C) \cup(C \cap \partial X)$. 
Proof of Lemma 2.12. Based on the local separation and the convexity of $F_{v}$, it is easy to check that $\bar{G}_{q i}, i=1,2$, are convex. By Theorem 2.13, we have that $F \cap \bar{G}_{q i} \subset \partial \bar{G}_{q i}$. Hence $[x y] \cap F=\emptyset$, because any minimal geodesic between interior points of a convex set does not intersect the boundary of the convex set $([24,5.2])$.

We are now in a position for the construction of a nonexpanding map.

For $x \in D$, let $q \in(S, a) \subset F_{u}, u \in N^{\prime}$, be a point such that $|x q|=|x F|$. First suppose $a \neq 0$. We will construct extended maximal gradient curves from $(S, a)$.

For $b \in(S, a)$, let $b_{j} \in G_{b 1}^{\circ}$, such that $b_{j} \rightarrow b$. Let $\gamma_{b}^{j}:\left[0, t_{0}\right] \rightarrow A, t_{0} \geq$ 0 denote the dist $_{F}$-gradient curve from $b_{j}$. Since $\gamma_{b}^{j}$ are equi-continuous, after passing to a subsequence, we can suppose that $\gamma_{b}^{j}$ converge to $\gamma_{b}:\left[0, t_{0}\right] \rightarrow A$ with $\gamma_{b}(0)=b$. Note that $\gamma_{b}$ doesn't depend on the choice of $b_{j}$. Indeed, for any $c \in U_{b} \cap(S, a)$, let $\gamma_{c}$ be a similarly constructed curve, namely, let $c_{j} \in G_{b 1}^{\circ}$, such that $c_{j} \rightarrow c$. The dist $_{F}$-gradient curve from $c_{j}, \gamma_{c}^{j}:\left[0, t_{0}\right] \rightarrow A$, after passing to a subsequence, converges to $\gamma_{c}:\left[0, t_{0}\right] \rightarrow A$ with $\gamma_{c}(0)=c$. Then $\left|\gamma_{b}(t) \gamma_{c}(t)\right|=$ $\lim _{j \rightarrow \infty}\left|\gamma_{b}^{j}(t) \gamma_{c}^{j}(t)\right|, 0 \leq t \leq t_{0}$. Since $\gamma_{b}^{j}(0), \gamma_{c}^{j}(0) \in G_{b 1}^{\circ} \subset U_{b}$, by Lemma 2.12, we have that $\left[\gamma_{b}^{j}(0) \gamma_{c}^{j}(0)\right] \cap F=\emptyset$. Set $\sigma(s):[0, l] \rightarrow\left[\gamma_{b}^{j}(0) \gamma_{c}^{j}(0)\right]$ with arc-length parametrization. Consider the curves $\sigma_{t}(s)=\Phi_{\operatorname{dist}_{F}}^{t}\left(\left[\gamma_{b}^{j}(0) \gamma_{c}^{j}(0)\right](s)\right)$, where $\Phi_{\text {dist }_{F}}^{t}$ is the standard dist $_{F}$-gradient flow defined in [30, 2.2]. Let $P_{m}=\left\{0=s_{0}<s_{1}<\right.$ $\left.\cdots<s_{m}=l, s_{i}-s_{i-1}=\frac{l}{m}\right\}$ be a partition of $[0, l]$, with $m$ large enough, such that $\frac{l}{m} \leq \frac{\varepsilon}{10}$, where $\varepsilon=\left|\left[\gamma_{b}^{j}(0) \gamma_{c}^{j}(0)\right], F\right|$. If $t \leq \frac{\varepsilon}{4}$, then $\left[\sigma_{t}\left(s_{i}\right) \sigma_{t}\left(s_{i-1}\right)\right] \cap F=$ $\emptyset$; thus $\left|\sigma_{t}\left(s_{i}\right) \sigma_{t}\left(s_{i-1}\right)\right| \leq\left|\sigma\left(s_{i}\right) \sigma\left(s_{i-1}\right)\right|$. Let $m \rightarrow \infty$; we get that $\left|\gamma_{b}^{j}(t) \gamma_{c}^{j}(t)\right| \leq$ Length $\left(\sigma_{t}\right) \leq \operatorname{Length}\left(\sigma_{0}\right)=\left|\gamma_{b}^{j}(0) \gamma_{c}^{j}(0)\right|$, where Length () denotes the length of the curve. If $t>\frac{\varepsilon}{4}$, we can repeat the procedure for $\left[\sigma_{\frac{\varepsilon}{4}}\left(s_{i}\right) \sigma_{\frac{\varepsilon}{4}}\left(s_{i-1}\right)\right]$. Finally, we get that for any $0 \leq t \leq t_{0},\left|\gamma_{b}^{j}(t) \gamma_{c}^{j}(t)\right| \leq\left|\gamma_{b}^{j}(0) \gamma_{c}^{j}(0)\right|$. When $j \rightarrow \infty$, we get that $\left|\gamma_{b}(t) \gamma_{c}(t)\right| \leq|b c|$. In particular, we have that if $b=c$, then $\gamma_{b}=\gamma_{c}$.

For simplicity, in the following context, we will say that $\gamma_{b}$ and $\gamma_{c}$ are in the same component with respect to $U_{b}$, and we will call $\gamma_{b}$ an extended maximal gradient curve if $\gamma_{b}-b$ is maximal.

By now we can see that for each point on $(S, a)$ there exist exactly two extended maximal gradient curves. Choose a point $y \in(S, a)$, and denote the two extended maximal gradient curves by $\gamma_{y 1}, \gamma_{y 0}$. For any other point, say $z$, we will denote the two extended maximal gradient curves from $z$ by $\gamma_{z 1}, \gamma_{z 0}$, such that $\gamma_{z i}$ are a continuation of $\gamma_{y i}$ along $[y z]$; i.e., there is a partition of $[y z], P=\left\{y_{0}=y, y_{1}, \cdots, y_{k}=z\right\}$, such that $\gamma_{y_{j}, i}, \gamma_{y_{j+1}, i}$ are in the same component with respect to $U_{y_{j}}$. It doesn't depend on the choice of the partition, since for another partition $P_{1}$, we can consider $P \cup P_{1}$ to get the independency.

Since $\pi_{1}((S, a))=0$, similarly as the final part of the proof of Key Lemma 0.7, we can see that the denoting doesn't depend on the choice of $[y z]$. Thus we can define two "flows" $\Psi_{i a}^{t}:(S, a) \rightarrow A$, by $b \rightarrow \gamma_{b i}(t), b \in(S, a)$, and we fix one passing $x$, which exists, since $[x q]$ is contained in an extended maximal gradient curve, denoted by $\Psi_{a}^{t}:(S, a) \rightarrow A$.

Lemma 2.14. The map $\Psi_{a}^{t}:(S, a) \rightarrow A$ is 1-Lipschitz.

Proof. For $b, c \in(S, a)$, we need to show that $|b c| \geq\left|\gamma_{b}(t) \gamma_{c}(t)\right|$. Dividing [bc] into small pieces so that the above local 1-Lipschitz property holds, one gets that $|b c| \geq \operatorname{Length}\left(\Psi_{a}^{t}([b c])\right) \geq\left|\Psi_{a}^{t}(b) \Psi_{a}^{t}(c)\right|$. 


\subsection{Completion of the proof of Theorem A.}

Lemma 2.15. Let $S_{x}=\Psi_{a}^{|x q|}((S, a))$. Then $\left.\pi\right|_{S_{x}}: S_{x} \rightarrow S$ is an isometry.

Proof. Let $i: S \rightarrow(S, a)$ denote the natural isometry. Define a map, $H:(S, a) \times$ $[0, l] \rightarrow(S, a)$, where $l=|x q|$, by $H(x, t)=i \circ \pi \circ \Psi_{a}^{t}(x)$. Since $\left|\Psi_{a}^{t}(x), \Psi_{a}^{t^{\prime}}\left(x^{\prime}\right)\right| \leq$ $\left|\Psi_{a}^{t}(x), \Psi_{a}^{t}\left(x^{\prime}\right)\right|+\left|\Psi_{a}^{t}\left(x^{\prime}\right), \Psi_{a}^{t^{\prime}}\left(x^{\prime}\right)\right| \leq\left|x x^{\prime}\right|+\left|t-t^{\prime}\right|, H$ is continuous. Since $H(*, 0)=$ $i d, H(x, l)$ is onto. Recall that given two 1-Lipschitz onto maps between two compact metric spaces, $g: X \rightarrow Y$ and $h: Y \rightarrow X$, then $g$ and $h$ are isometries. Since $\Psi_{a}^{t}$ and $\pi$ are 1-Lipschitz, the desired result follows.

If $a=0$, consider $\Sigma_{q} A$. From the proof of Lemma 2.8, we get that there are $u, v \in \phi_{[p q]}\left(N^{\prime}\right)\left(u\right.$ may be equal to $v$ ) such that $\left|u \uparrow_{q}^{x}\right|=\left|v \uparrow_{q}^{x}\right|=\frac{\pi}{2}$. Let $x_{i} \in[x q], y_{i} \in\left(\left(S, a_{i}\right) \cap \pi^{-1}(q)\right) \subset F_{\phi_{[p q]}^{-1}(u)}$, where $a_{i} \neq 0$, and $x_{i} \rightarrow q, y_{i} \rightarrow q$, as $i \rightarrow \infty$.

Claim 2.16. There exist $\bar{y}_{i} \in\left[x_{i} y_{i}\right] \cap F$ and $\bar{y}_{i} \notin S$ such that $\left[x_{i} \bar{y}_{i}\right]^{\circ} \cap F=\emptyset$.

Suppose that $\bar{y}_{i} \in\left(S, b_{i}\right)$ with $b_{i} \neq 0$. Let $\Psi_{b_{i}}^{t}:\left(S, b_{i}\right) \rightarrow A$ denote the "flow" such that $\Psi_{b_{i}}^{t}\left(\bar{y}_{i}\right), t \leq \varepsilon$ ( $\varepsilon$ small enough), is in the same component (defined after Lemma 2.11) as $\left[y_{i}^{\prime} \bar{y}_{i}\right] \subset\left[x_{i} \bar{y}_{i}\right]$ with $y_{i}^{\prime}, \bar{y}_{i}$ close enough, and let $\gamma_{i}(t)=\Psi_{b_{i}}^{t}\left(\bar{y}_{i}\right)$. Then by the construction of $\gamma_{i}(t)$, one obtains that $\left|\gamma_{i}(t),\left[x_{i} x\right](t)\right| \leq\left|\bar{y}_{i} x_{i}\right|$; thus $\gamma_{i}(t) \rightarrow[x q](t)$, for any $0 \leq t \leq|x q|$, as $i \rightarrow \infty$. Hence by passing to a subsequence we can suppose that $\Psi_{b_{i}}^{|x q|}\left(\left(S, b_{i}\right)\right) \stackrel{\text { isom }}{\cong} S$ converge to $S_{x} \stackrel{\text { isom }}{\cong} S$, with $x \in S_{x}$. Since $\left.\pi\right|_{\Psi_{b_{i}}^{|x q|}\left(\left(S, b_{i}\right)\right)}$ is an isometry, so is $\left.\pi\right|_{S_{x}}$.

Proof of Claim 2.16. It suffices to show that if $z \in\left[x_{i} y_{i}\right] \cap F$, then $z \notin S$. Argue by contradiction and suppose $z \in S$. Since $\left|x_{i} q\right| \leq\left|x_{i} z\right|$ and $\left|y_{i} q\right| \leq\left|y_{i} z\right|$, we have that $\left[x_{i} q\right] \cup\left[q y_{i}\right]$ is a minimal geodesic, which is impossible, because by the choice of $y_{i}$ we have that $\uparrow_{q}^{x_{i}} \perp \uparrow_{q}^{y_{i}}$.

Now we are ready for the proof of Theorem A.

Proof of Theorem A.

Case 1. $\mathscr{F}=A$. See the proof following Lemma 1.1.

Case 2. $\mathscr{F} \neq A$. By Lemma 1.5, we can assume that $\pi_{1}(S)=0$. For $x \in A$ and for any $\bar{y} \in S$, let $y=S_{x} \cap \pi^{-1}(\bar{y})$. Since $\left.\pi\right|_{S_{x}}: S_{x} \rightarrow S$ is an isometry, $|x y|=|\bar{x} \bar{y}|$, and this shows that $\pi: A \rightarrow S$ is a submetry.

\section{Application}

We will prove Corollary 0.6, and we will show that in Theorem $\mathrm{A}$ if $\mathscr{F}=A$, then $\pi$ is a bundle map. First we recall the following lemma.

Lemma 3.1. Let $A \in \operatorname{Alex}^{4}(\kappa)$. Then the following statements are equivalent:

(3.1.1) A is topologically nice.

(3.1.2) A is topologically regular.

(3.1.3) A is a topological manifold.

Lemma 3.1 is pointed out by Kapovitch in [21]; for completeness we include a proof here. 
Proof. It is obvious that (3.1.1) $\Rightarrow(3.1 .2)$ and (3.1.2) $\Rightarrow(3.1 .3)$.

$(3.1 .3) \Rightarrow(3.1 .1)$ : Any manifold point $p \in A$ satisfies that $\pi_{1}\left(\Sigma_{p} A\right)=0$ (cf. [41, Theorem $1.1(2)]$ ) and that $H_{*}\left(\Sigma_{v} \Sigma_{p} A\right)=H_{*}\left(S^{2}\right)$ ([41, Proposition 3.1]). Hence $\Sigma_{v} \Sigma_{p} A$ is an $S^{2}$, and therefore $\Sigma_{p} A$ is a simply connected manifold. By the 3 -dimension Poincaré conjecture, $\Sigma_{p} A$ is homeomorphic to $S^{3}$, and thus $A$ is topologically nice.

Proof of Corollary 0.6. Argue by contradiction and suppose that $\operatorname{dim}(S)>0$. By 38, 9.8], $\operatorname{dim}(S) \neq 1,3$. If $\operatorname{dim}(S)=2$, we may assume that $q \in A$ such that the curvature is positive in $B(q, r)$. Then the modified Busemann function - $\exp ^{f}$ is strictly concave $([8])$, and thus $\left.\pi\right|_{B(q, r)}: B(q, r) \rightarrow B(\pi(q), r)$ is strictly distance decreasing, a contradiction to Theorem A.

Corollary 3.2. Let the assumptions be as in Theorem A. Suppose $\mathscr{F}=A$. Then $\pi: A \rightarrow S$ is a bundle map with fiber $\mathbb{R}^{2}$.

Note that Corollary 3.2 doesn't hold if one removes the condition of topologically nice; see [42, 14.8].

In the proof, we shall apply the following theorem, which is a sufficient condition for a bundle map:

Theorem 3.3 (33]). Let $X, X_{0}$ be two metric spaces and let $f: X \rightarrow X_{0}$ be a continuous onto map. Suppose that for any $p \in X_{0}$ and $\varepsilon>0$ there exists a $\delta(p, \varepsilon)>0$ such that for any $q \in B(p, \delta)$, there is a homeomorphism $h: f^{-1}(p) \rightarrow$ $f^{-1}(q)$, with $|h(x) x| \leq \varepsilon$, for any $x \in f^{-1}(p)$. If the fiber $F$ is locally compact and separable, and the homeomorphism group of $F$ (with some natural topology) is locally path connected, then $F$ is a Serre fibration. If, in addition, $X_{0}$ is finite dimensional ANR, then $f$ is a locally trivial bundle map.

Proof of Corollary 3.2. Since $\mathscr{F}=A$, for any $p, q \in S$ and a fixed $[p q]$, there is a homeomorphism $h: \pi^{-1}(p) \rightarrow \pi^{-1}(q)$ such that $[x p],[p q],[q h(x)]$ determine a unique flat rectangle (Lemma (1.1.2) and Proposition (2.1.3)). Then $|h(x) x|=|p q|$.

Since $A$ is topologically nice and $\pi$ is a submetry, if $p \in S$ is a regular point (i.e., $T_{p} S$ is isometric to $\mathbb{R}^{n-2}$ ), then $\pi^{-1}(p)$ is a topological manifold ([32, Theorem $\mathrm{D}]$; note that the proof is local and thus applies to noncompact cases). Since $\pi: \pi^{-1}(p) \rightarrow p$ is a deformation retraction, $\pi^{-1}(p) \stackrel{\text { homeo }}{\simeq} \mathbb{R}^{2}$.

Also, by [11, 7.3], we know that the homeomorphism group homeo $\left(\pi^{-1}(p)\right)$ is locally path connected with the topology in Theorem 3.3. By now we are able to apply Theorem 3.3 to conclude that $\pi$ is a bundle map.

Remark 3.4. Inspired by [40, it seems that for Case 2 of Theorem A, when $\pi_{1}(A)=$ $0, A$ isometrically splits.

\section{Structure of SPaCe of Directions}

Our main effort in this section is to prove Proposition 2.1 and thus complete the proof of Theorem A. We point out that Theorem 4.4, which classifies certain isometric classes in $\operatorname{Alex}^{n}(1)$, may have independent interest.

First we recall the following:

Definition 4.1 ([3]). Let $X, Y \in \operatorname{Alex}(1)$. The join of $X$ and $Y, X * Y$, is defined by $X \times Y \times\left[0, \frac{\pi}{2}\right] / \sim$, where $\left(x, y_{1}, 0\right) \sim\left(x, y_{2}, 0\right)$ and $\left(x_{1}, y, \frac{\pi}{2}\right) \sim\left(x_{2}, y, \frac{\pi}{2}\right)$, with the metric: $\cos d\left(\left(x_{1}, y_{1}, t\right),\left(x_{2}, y_{2}, s\right)\right)=\cos t \cos s \cos \left(\left|x_{1} x_{2}\right|\right)+\sin t \sin s \cos \left(\left|y_{1} y_{2}\right|\right)$. 
Then $X * Y \in \operatorname{Alex}(1)$ and $C(X * Y)=C(X) \times C(Y)([3])$.

Example 4.2. $S^{m} * S^{n}=S^{m+n+1}$, where all the spheres are with the standard metric with constant curvature 1 .

Remark 4.3. For the convenience of following use, we will make the following conventions:

(4.3.1) Let 0-dim Alexandrov space with curvature bounded below by 1 without boundary be a space including two points with distance $\pi$; one point is regraded as with boundary.

(4.3.2) If a subspace with restricted metric is isometric to an Alexandrov space, we also say that it is convex, although when the dimension is 0 , there may not be a minimal geodesic in the subspace joining two given points.

(4.3.3) When we say that two metric spaces are equal, we always mean metrically, except when otherwise stated. If there is no confusion, we will not mention the metric.

Let $A \in \operatorname{Alex}^{n}(1)$ and let $C \subset A$ be a closed convex subset without boundary. Let $\hat{\Sigma}_{p} C=\left\{v \in \Sigma_{p} A|| v \Sigma_{p} C \mid \geq \frac{\pi}{2}\right\}$, and let $\hat{T}_{p} C=C\left(\hat{\Sigma}_{p} C\right)$. Observe that if $A$ and $C$ are Riemannian manifolds, then $T_{p} A$ isometrically splits, i.e., $T_{p} A=T_{p} C \times \hat{T}_{p} C$ or equivalently $\Sigma_{p} A$ is isometric to the join of $\Sigma_{p} C$ and $\hat{\Sigma}_{p} C$ ([3]). In Alexandrov geometry, such a property doesn't hold.

Observe that for $\Sigma \in \operatorname{Alex}^{n}(1)$ and $\Sigma_{0}, \Sigma_{1} \subset \Sigma$ convex closed subsets, if $\Sigma=$ $\Sigma_{0} * \Sigma_{1}$, then $\Sigma_{0}, \Sigma_{1}$ satisfy the following two conditions:

(a) For every $v \in \Sigma, v \in\left[v_{0} v_{1}\right]$, for some $v_{0} \in \Sigma_{0}, v_{1} \in \Sigma_{1}$.

(b) $\operatorname{dim}\left(\Sigma_{0}\right)+\operatorname{dim}\left(\Sigma_{1}\right)+1=n$ (dim denotes the Hausdorff dimension).

In the following situation, conditions (a) and (b) are indeed sufficient conditions for a join.

Theorem 4.4. Let $\Sigma \in \operatorname{Alex}^{n}(1)$, let $\Sigma_{0} \subset \Sigma$ be a closed convex subset without boundary, and let $\Sigma_{1}=\left\{v \in \Sigma|| v \Sigma_{0} \mid \geq \frac{\pi}{2}\right\}$. Assume that $\Sigma_{1} \neq \emptyset$ and $\Sigma_{0}, \Sigma_{1}$ satisfy conditions (a) and (b). If $\Sigma$ is topologically nice and homeomorphic to a sphere, then $\Sigma=\Sigma_{0} * \Sigma_{1}$ and $\Sigma_{i}, i=0,1$, are topologically nice and homeomorphic to spheres.

Theorem 4.4 is related to and was inspired by the following conjecture made by Yamaguchi [42, 14.6].

Conjecture 4.5. Let $A \in \operatorname{Alex}^{n}(0)$ be noncompact with a soul $S$. If $p \in\left(C_{i}-\partial C_{i}\right)$ is a topologically regular point, then $T_{p} X$ is isometric to the product $T_{p} C_{i} \times K$, where $C_{i}$ are as in section 1.1, and $K$ is a Euclidean cone.

Yamaguchi proved Conjecture 4.5 for $n=4$ ([42, Theorem 14.5]), where topologically nice and topologically regular are equivalent.

Using Theorem 4.4, with an additional argument, we can get the following classification result, which can imply Proposition 2.1 .

Theorem 4.6. Let $\Sigma \in \operatorname{Alex}^{n}(1), n \geq 2$. Let $\Sigma_{0} \subset \Sigma$ be a convex closed subset without boundary with dimension $n-2$, and let $\Sigma_{1}=\left\{u \in \Sigma|| u \Sigma_{0} \mid \geq \frac{\pi}{2}\right\}$. Suppose that $\Sigma_{1} \neq \emptyset, \Sigma$ is homeomorphic to a sphere and topologically nice, and $\Sigma$ satisfies that for any $u \in \Sigma,\left|u \Sigma_{0}\right| \leq \frac{\pi}{2}$ and $\left|u \Sigma_{1}\right| \leq \frac{\pi}{2}$. Then $\Sigma_{1}$ is convex, $\Sigma_{0}$ is homeomorphic to $S^{n-2}$ and topologically nice, and $\left[\Sigma_{0} \Sigma_{1}\right]=\Sigma_{0} * \Sigma_{1}$, where $\left[\Sigma_{0} \Sigma_{1}\right]$ 
is with the restricted metric. Explicitly, we get the following classifications:

(1) If $\Sigma_{1}=S^{1}(r)$ with $r \leq 1$, then $\Sigma=\Sigma_{0} * \Sigma_{1}$.

(2) If $\Sigma_{1}=\left\{v_{1}, v_{2}\right\}$ with $\left|v_{1} v_{2}\right|=\pi$, then $\Sigma=\Sigma_{1} * \Sigma_{1}^{\perp}$.

(3) If $\Sigma_{1}=\{v\}$, then $\Sigma=D\left(\Sigma_{1} * \hat{\Sigma}_{1}\right)$, the double of half suspension of $\hat{\Sigma}_{1}=$ $\left\{u \in \Sigma|| u \Sigma_{1} \mid \geq \frac{\pi}{2}\right\}$.

(4) If $\Sigma_{1}=[a b]$, then $\Sigma=\left(a * \hat{\Sigma}_{1} \cup_{\hat{\Sigma}_{1}} b * \hat{\Sigma}_{1}\right) \cup_{\partial}\left(\Sigma_{1} * \Sigma_{0}\right)$ with the gluing metric ([2, Definition 3.1.12]).

In the rest of this section, we will prove Theorem 4.4 and Theorem 4.6. First we will present some preparations.

Lemma 4.7. Let $\Sigma \in \operatorname{Alex}^{n}(1)$ and let $\Sigma_{1}, \Sigma_{0} \subset \Sigma$ be two convex closed subsets with dimension $k, l$ respectively. Suppose that $\Sigma$ satisfies: for any $v_{0}, v_{1} \in \Sigma_{0}, \Sigma_{1}$ respectively, $\left|v_{0} v_{1}\right|=\frac{\pi}{2}$. Then $n \geq l+k+1$.

Proof. Let $C_{i} \subset \Sigma_{i} \backslash \partial \Sigma_{i}, i=1,0$, be two closed convex subsets. Note that $\left[C_{1} C_{0}\right]$ with the restricted metric is a closed subset of $\Sigma$. It suffices to construct a nonexpanding map from $\left[C_{1} C_{0}\right]$ to $C_{1} * C_{0}$. If so, $\operatorname{dim}(\Sigma) \geq \operatorname{dim}\left(\left[C_{1} C_{0}\right]\right) \geq$ $\operatorname{dim}\left(C_{1} * C_{0}\right)=k+l+1$, where the last identity is because, by definition, we have that $\operatorname{dim}\left(C\left(C_{0} * C_{1}\right)\right)=\operatorname{dim}\left(C\left(C_{0}\right) \times C\left(C_{1}\right)\right)=k+l+2$.

First we claim that $\left[x_{1} y_{1}\right] \cap\left[x_{2} y_{2}\right] \subset\left\{x_{1}, y_{1}, x_{2}, y_{2}\right\}$, for $x_{1}, x_{2} \in C_{1}, y_{1}, y_{2} \in C_{0}$. Thus for $p \in\left[C_{1} C_{0}\right], p$ can be uniquely written as $([x y], s)$, where $x \in C_{1}, y \in$ $C_{0}, p \in[x y]$ and $s=|p x|$. Then we can construct a map, $g:\left[C_{1} C_{0}\right] \rightarrow C_{1} * C_{0}$, by $\left.g\right|_{C_{i}}=i d, i=0,1$ and $g(([p q], s))=([p q], s)$ (the unique point on the unique minimal geodesic $[p q]$ with distance $s$ from $p$ ), for $p \in C_{1}, q \in C_{0}$.

Next we will show that $g$ is nonexpanding. In order to do so we need the following fact:

For $p \in C_{1}$ and $q \in C_{0},|p q|=\left|p \Sigma_{0}\right|$. By Lemma 1.2, $\left|\uparrow_{q}^{p} v\right|=\frac{\pi}{2}$, for any $v \in \Sigma_{q} \Sigma_{0}$. Hence $\tilde{L}(p, q, r)=\angle(p, q, r)=\frac{\pi}{2}$, for any $r \in \Sigma_{0}$. By Lemma 2.6, there is a convex triangle isometric to the corresponding one on space form.

For $x, y \in\left[C_{1} C_{0}\right]$, by the property of $\left[C_{1} C_{0}\right]$, we have that $x \in\left[p_{1} p_{0}\right], y \in\left[q_{1} q_{0}\right]$. Let $\left|x p_{1}\right|=s$ and $\left|y q_{1}\right|=t$. Then by the above fact, $\cos \left(\left|p_{1} y\right|\right)=\cos \left(\left|p_{1} q_{1}\right|\right) \cos t$, $\cos \left(\left|p_{0} y\right|\right)=\cos \left(\left|p_{0} q_{0}\right|\right) \sin t$. By the monotonicity of angle,

$$
|x y| \geq \cos s \cos t \cos \left(\left|p_{1} q_{1}\right|\right)+\sin s \sin t \cos \left(\left|p_{0} q_{0}\right|\right)=|g(x) g(y)|,
$$

which shows that $g$ is nonexpanding.

Finally, we will verify the claim. It suffices to show that if $x_{1} \neq x_{2}, y_{1} \neq$ $y_{2}$, then $\left[x_{1} y_{1}\right] \cap\left[x_{2} y_{2}\right]=\emptyset$. Suppose that $\left[x_{1} y_{1}\right] \cap\left[x_{2} y_{2}\right]=z$. Hence by the above fact, $\left[z y_{1}\right] \subset$ the totally geodesic triangle bounded by $\left\{x_{2}, y_{1}, y_{2}\right\}$, which is isometric to the corresponding triangle on $S^{2}(1)$, or the geodesic will branch. Thus $\angle\left(x_{1}, y_{1}, y_{2}\right)<\frac{\pi}{2}$. This is a contradiction.

Remark 4.8. Let $\Sigma \in \operatorname{Alex}^{n}(1)$, and let $\Sigma_{0} \subset \Sigma$ be a convex closed subset. Suppose $\Sigma_{1}=\left\{v \in \Sigma|| v \Sigma_{0} \mid \geq \frac{\pi}{2}\right\} \neq \emptyset$. If $\partial \Sigma_{0}=\emptyset$ or $\partial \Sigma_{1}=\emptyset$, then by Lemma 1.2, $\Sigma$ satisfies the conditions of Lemma 4.7. These are the cases usually used in the following context.

4.1. Proof of Theorem 4.4. Sketch of the proof. We will prove Theorem 4.4 by induction on the dimension of $\Sigma$, and we will prove the inductive step according to the different situations of the dimensions of $\Sigma_{0}$ and $\Sigma_{1}$. Except for two simple cases, we will first show that for each point in $\Sigma_{1}$ and each point in $\Sigma_{0}$, there exist exactly 
$m$ minimal geodesics joining them for some $m>0$. Then arguing by contradiction, we can get that $m=1$, and using this we can derive that $\Sigma$ is a join.

Proof of Theorem 4.4. Denote $\operatorname{dim}\left(\Sigma_{1}\right)$ by $I$ and $\operatorname{dim}\left(\Sigma_{0}\right)$ by $J$. First we will prove the following property.

Sublemma 4.9. We have that $\Sigma_{1}$ is convex.

Proof. For $p, q \in \Sigma_{1}$, when $|p q|<\pi$, by triangle comparison we have that for any $x \in \Sigma_{0},|x,[p q]| \geq \frac{\pi}{2}$. Hence $[p q] \subset \Sigma_{1}$. When $|p q|=\pi$, if there are no other points in $\Sigma_{1}$, then the sublemma holds. If there is $x \in \Sigma_{1}-\{p, q\}$, one can easily see that $|p x|+|x q|=\pi=|p q|$; thus $[p x],[x q] \subset \Sigma_{1}$, and $[p x] \cup[x q]$ is a minimal geodesic which lies in $\Sigma_{1}$. The sublemma thus follows.

We then proceed with the proof by induction on $n$. For $n=1$, i.e., $I=0$ and $J=0$, clearly the theorem holds. Suppose the theorem holds for $n-1$.

Now we will prove the inductive step according to different situations of $I$ and $J$ :

If $J=0$, i.e., $\Sigma_{0}=\left\{v_{1}, v_{2}\right\}$ with $\left|v_{1} v_{2}\right|=\pi$, then $\Sigma=\Sigma_{0} * \Sigma_{1}$. Thus $\Sigma_{v_{1}}=\Sigma_{1}$. Because $\Sigma$ is topologically nice, $\Sigma_{1}$ is homeomorphic to $S^{n-1}$ and topologically nice.

Hence in the following we can assume that $J>0$.

Next we want to show that $\partial \Sigma_{1}=\emptyset$. In order to apply inductive assumptions to $\Sigma_{p} \Sigma$, for $p \in \Sigma_{0}$, it suffices to check that $\Sigma_{p} \Sigma_{0},\left(\Sigma_{p} \Sigma_{0}\right)^{\perp}$ satisfy conditions (a) and (b). For condition (a):

Sublemma 4.10. For any $p \in \Sigma_{0}$ and for any $\bar{v} \in \Sigma_{p} \Sigma, \bar{v} \in\left[\bar{v}_{1} \bar{v}_{0}\right] \subset\left[\Uparrow_{p}^{\Sigma_{1}} \Sigma_{p} \Sigma_{0}\right]$.

Sublemma 4.10 implies that for $p \in \Sigma_{0},\left(\Sigma_{p} \Sigma_{0}\right)^{\perp}=\Uparrow_{p}^{\Sigma_{1}}$ with the restricted metric in $\Sigma_{p}$.

Proof of Sublemma 4.10. Observe that by Lemma 1.2, we have that for every $p \in$ $\Sigma_{1}$ and every $q \in \Sigma_{0},|p q|=\frac{\pi}{2}$. Hence for every $r \in \Sigma_{0}, \angle(p, q, r)=\tilde{L}(p, q, r)$. By Lemma 2.6, we have that there exists a totally geodesic triangle determined by $[p q]$ and $[q r]$, which is isometric to a geodesic triangle in $S^{2}(1)$.

Let $\bar{v} \in\left(\Sigma_{p} \Sigma\right)^{\prime}$. Then $\bar{v}=\uparrow_{p}^{x}$ for some $x \in \Sigma$. By perturbing $x$ along $[p x]$, we can suppose that $[p x]$ is unique. From condition (a), $x \in\left[x_{1} x_{0}\right]$, for some $x_{i} \in$ $\Sigma_{i}$. Therefore there exists a totally geodesic triangle $T$ determined by $\left[x_{0} p\right],\left[x_{1} p\right]$, which is isometric to a geodesic triangle in $S^{2}(1)$, and by the uniqueness, we get that $[p x] \subset T$. Thus $\bar{v} \in\left[\uparrow_{p}^{x_{1} \uparrow_{p} x_{0}}\right]$. Since $\left(\Sigma_{p} \Sigma\right)^{\prime}$ is dense in $\Sigma_{p} \Sigma$, we get the sublemma.

For condition (b): From the construction, we see that $\psi_{1}: \Uparrow_{p}^{\Sigma_{1}} \rightarrow \Sigma_{1}, \psi_{1}\left(\Uparrow_{p}^{x}\right)=$ $x, x \in \Sigma_{1}$, is a submetry (since $\psi_{1}$ has the horizontal lifting property, as can be seen in the proof of Sublemma 4.10). Thus the map $\psi_{1}$ is 1-Lipschitz. Therefore $\operatorname{dim}\left(\Uparrow_{p}^{\Sigma_{1}}\right) \geq \operatorname{dim}\left(\Sigma_{1}\right)$. Hence on one hand, we have

$$
\operatorname{dim}\left(\Sigma_{p} \Sigma\right)=\operatorname{dim}\left(\Sigma_{0}\right)-1+\operatorname{dim}\left(\Sigma_{1}\right)+1 \leq \operatorname{dim}\left(\Sigma_{p} \Sigma_{0}\right)+\operatorname{dim}\left(\Uparrow_{p}^{\Sigma_{1}}\right)+1 .
$$

On the other hand, by Lemma 4.7, we have the opposite inequality. Thus

$$
\operatorname{dim}\left(\Sigma_{p} \Sigma\right)=\operatorname{dim}\left(\Sigma_{p} \Sigma_{0}\right)+\operatorname{dim}\left(\Uparrow_{p}^{\Sigma_{1}}\right)+1 .
$$


By now, we obtain that $\Sigma_{p} \Sigma, p \in \Sigma_{0}$, satisfies the inductive assumptions. Hence for every $p \in \Sigma_{0}, \Sigma_{p} \Sigma=\Sigma_{p} \Sigma_{0} *\left(\Sigma_{p} \Sigma_{0}\right)^{\perp}$, and $\Sigma_{p} \Sigma_{0}$ is homeomorphic to a sphere. Thus $\Sigma_{0}$ is a topological manifold.

For the following use, we recall Alexander duality [19]: Let $K \subset S^{n}$ be a compact, locally contractible, nonempty, proper subspace. Then $\widetilde{H}_{i}\left(S^{n}-K ; \mathbb{Z}\right)=$ $\widetilde{H}^{n-i-1}(K ; \mathbb{Z})$, for all $i$, where $\widetilde{H}_{i}, \widetilde{H}^{i}$ denote the reduced homology and cohomology.

Now we are ready to show that $\partial \Sigma_{1}=\emptyset$. Arguing by contradiction, assume $\partial \Sigma_{1} \neq \emptyset$. Then $\Sigma_{1}$ is contractible, since $\Sigma_{1}$ has positive curvature (cf. 24]). From condition (1), we see that dist $\Sigma_{1}$ has no critical points in $\Sigma-\Sigma_{1} \cup \Sigma_{0}$. Thus we have that there is a deformation retraction from $\Sigma-\Sigma_{1}$ to $\Sigma_{0}$. Therefore $\widetilde{H}^{n-1-i}\left(\Sigma_{1}\right)=$ 0 . On one hand, by Alexander duality $\widetilde{H}_{i}\left(S^{n}-\Sigma_{1}\right)=\widetilde{H}_{i}\left(\Sigma_{0}\right)=\widetilde{H}^{n-1-i}\left(\Sigma_{1}\right)=0$, for any $i \geq 0$. On the other hand, since $\Sigma_{0}$ is a topological manifold, it follows that if $\Sigma_{0}$ is orientable, then $\widetilde{H}_{n}\left(\Sigma_{0}\right) \neq 0$, and if $\Sigma_{0}$ is not orientable, then $\widetilde{H}_{n-1}\left(\Sigma_{0}\right) \neq 0$. This is a contradiction. Therefore $\partial \Sigma_{1}=\emptyset$.

Now we come to the second case:

If $I=0$, we have that $\Sigma_{1}=\left\{w_{1}, w_{2}\right\}$ with $\left|w_{1} w_{2}\right|=\pi$. Then $\Sigma=\Sigma_{0} * \Sigma_{1}$. Because $\Sigma$ is topologically nice, $\Sigma_{0}$ is homeomorphic to $S^{n-1}$ and topologically nice.

Hence in the following we can suppose that $I>0$. First we will show that for any $x_{i} \in \Sigma_{i}, i=0,1$, there are $m$ minimal geodesics joining $x_{0}$ and $x_{1}$, for some $m>0$.

Similarly, we have that for every $x \in \Sigma_{1}$,

$$
\operatorname{dim}\left(\Sigma_{x} \Sigma\right)=\operatorname{dim}\left(\Sigma_{x} \Sigma_{1}\right)+\operatorname{dim}\left(\Uparrow_{x}^{\Sigma_{0}}\right)+1 .
$$

Hence $\operatorname{dim}\left(\Uparrow_{x}^{\Sigma_{0}}\right)=\operatorname{dim}\left(\Sigma_{0}\right)$.

Since $\partial \Sigma_{1}=\emptyset$, we can apply Sublemma 4.10 to $\Sigma_{x} \Sigma, x \in \Sigma_{1}$. We get that $\Sigma_{x} \Sigma_{1},\left(\Sigma_{x} \Sigma_{1}\right)^{\perp}$ satisfy condition (a) and $\Uparrow_{x}^{\Sigma_{0}}=\left(\Sigma_{x} \Sigma_{1}\right)^{\perp}$. Hence $\Uparrow_{x}^{\Sigma_{0}}$ is convex, and similarly, $\psi_{0}: \uparrow_{x}^{\Sigma_{0}} \rightarrow \Sigma_{0}, \psi_{0}\left(\Uparrow_{x}^{p}\right)=p, p \in \Sigma_{0}$, is a submetry. Thus for a $(J, \delta)$-burst point (see [3, 5.2]) $y \in \Sigma_{0}$, there is a neighborhood $U_{y}$ of $y$ such that $\psi_{0}^{-1}\left(U_{y}\right) \stackrel{\text { homeo }}{\simeq}$ $U_{y} \times F_{0}$, where $F_{0}$ is a $0-\operatorname{dim} M C S$-space $\left.(24]\right)$. Hence $F_{0}$ is a collection of discrete points. Since $\Uparrow_{x}^{\Sigma_{0}}$ is compact, $\#\left\{\psi_{0}^{-1}(y)\right\}<\infty$, say $m$. In the following, we will show that $m$ is independent of the choice of $y$.

Sublemma 4.11. For every $x \in \Sigma_{1}$ and every $p, q \in \Sigma_{0}$, $\#\left\{\Uparrow_{x}^{p}\right\}=\#\left\{\Uparrow_{x}^{q}\right\}$.

Proof. Since for a fixed minimal geodesic $[p q]$ and for any $[x p]$, there is a totally geodesic triangle with two sides $[p q],[x p]$ which is isometric to a geodesic triangle in $S^{2}(1)$, there is a corresponding $[x q]$. This determines a map from $\Uparrow_{x}^{p}$ to $\Uparrow_{x}^{q}$ which is 1-1 onto, or there will be a contradiction to the join of $\Sigma_{p}$ or $\Sigma_{q}$, and thus the sublemma follows.

Then we want to prove the following:

Sublemma 4.12. The map $\psi_{0}$ is an $m$-fold locally isometric covering map.

Proof. Let $\psi_{0}^{-1}(p)=\bigcup_{i=1, \cdots, m}\left\{p_{i}\right\}$ and $\varepsilon=\min _{1 \leq i, j \leq m}\left\{\left|p_{i} p_{j}\right|\right\}$. Then $B\left(p_{i}, \frac{\varepsilon}{5}\right) \cap$ $B\left(p_{j}, \frac{\varepsilon}{5}\right)=\emptyset$, since if there is $x \in B\left(p_{i}, \frac{\varepsilon}{5}\right) \cap B\left(p_{j}, \frac{\varepsilon}{5}\right)$, then $\left|p_{i} p_{j}\right| \leq\left|x p_{i}\right|+\left|x p_{j}\right| \leq \frac{\varepsilon}{2}$, a contradiction. Hence $\psi_{0}^{-1}\left(B\left(p, \frac{\varepsilon}{5}\right)\right)=B\left(\psi_{0}^{-1}(p), \frac{\varepsilon}{5}\right)=B\left(\cup\left\{p_{i}\right\}, \frac{\varepsilon}{5}\right)=\cup B\left(p_{i}, \frac{\varepsilon}{5}\right)$. 
Next we will show that $B\left(p, \frac{\varepsilon}{5}\right)$ is isometric to $B\left(p_{i}, \frac{\varepsilon}{5}\right)$, for any $1 \leq i \leq m$. For any $\bar{x} \in B\left(p, \frac{\varepsilon}{5}\right)$, there exists a unique $x \in B\left(p_{i}, \frac{\varepsilon}{5}\right)$ such that $\left|p_{i} x\right|=|p \bar{x}|$, or it will contradict Sublemma 4.11. For $\bar{y} \in B\left(p, \frac{\varepsilon}{5}\right)$, let $y \in B\left(p_{i}, \frac{\varepsilon}{5}\right)$ such that $|y x|=|\bar{x} \bar{y}|$. Then $\left|p_{i} y\right|=|p \bar{y}|$, or $\left|p_{i} p_{j}\right| \leq \frac{4 \varepsilon}{5}$, a contradiction. Thus we get the sublemma.

Since we have showed that for any $x \in \Sigma_{1}, \Sigma_{x} \Sigma$ satisfies the conditions of Theorem 4.4, by induction assumption, $\Sigma_{x} \Sigma=\Sigma_{x} \Sigma_{1} * \Uparrow_{x}^{\Sigma_{0}}$ and $\Sigma_{x} \Sigma_{1}$, $\Uparrow_{x}^{\Sigma_{0}}$ are homeomorphic to spheres.

Hence $\Sigma_{0} \stackrel{\text { homeo }}{\simeq} S^{J} / \Gamma_{0}$. Similarly, we get that for any $p \in \Sigma_{0}, \psi_{1}: \uparrow_{p}^{\Sigma_{1}} \rightarrow \Sigma_{1}$ is an $m$-fold covering map, and therefore $\Sigma_{1} \stackrel{\text { homeo }}{\simeq} S^{I} / \Gamma_{1}$. By now we can see that for any $x_{i} \in \Sigma_{i}$, there are $m$ minimal geodesics joining $x_{0}$ and $x_{1}$.

Next, we want to show that $m=1$.

Sublemma 4.13. Let $X_{i}=\left\{v|| v \Sigma_{i} \mid \leq \frac{\pi}{4}\right\}, i=0,1$. Then $X_{i}$ are locally trivial bundles over $\Sigma_{i}$.

Proof. Without loss of generality, we can suppose that $i=0$. First observe that there is a natural map, $g: X_{0} \rightarrow \Sigma_{0}$, defined in the following way: for any $y \in X_{0}$, by condition (a), we have that $y \in\left[y_{1} y_{0}\right]$, for some $y_{i} \in \Sigma_{i}$; set $g(y)=y_{0}$.

For $p \in \Sigma_{0}$, let $U$ be a locally isometric neighborhood of $p$ as in Sublemma 4.12. Then $U$ satisfies that the covering map $\psi_{0}: \uparrow_{x}^{\Sigma_{0}} \rightarrow \Sigma_{0}$, for any $x \in \Sigma_{1}$, when restricted to each component of $\psi_{0}^{-1}(U)$, is an isometry. Indeed, if $\Sigma_{0}$ is an $S^{1}$, then nothing need be said. If $\Sigma_{0}$ is not an $S^{1}$, let $Y_{1}, Y_{2}$ be two universal covering spaces of $\Sigma_{0}$, with covering maps $c_{1}, c_{2}$. There exists an isometry, $g: Y_{1} \rightarrow Y_{2}$, which is fiber preserving, i.e., $c_{2} \circ g=c_{1}$. Then we can get a neighborhood $V$ such that if $c_{1}$, when restricted to $c_{1}^{-1}(V)$, is an isometry; thus so is $c_{2}$.

Now we can define a map, $\eta: g^{-1}(U) \rightarrow U \times F$, where $F=g^{-1}(p)$, in the following way: for $z \in g^{-1}(U)$, then $z \in\left[z_{1} z_{0}\right]$ for some $z_{i} \in \Sigma_{i}$, set $\eta(z)=$ $\left(z_{0},\left(\left[z_{1} p\right],\left|z z_{1}\right|\right)\right)$, where $\left(\left[z_{1} p\right],\left|z z_{1}\right|\right)$ represents a point on the geodesic $\left[z_{1} p\right]$ with distance $\left|z z_{1}\right|$ from $z_{1}$, and $\left[z_{1} p\right]$ is the geodesic such that $\left[\uparrow_{z_{1}} \uparrow z_{z_{1}}\right]$ is the horizontal lifting of $\left[p z_{0}\right]$, i.e., $\left|\uparrow z_{1} \uparrow z_{z_{1}}\right|=\left|p z_{0}\right|$, which is unique, by the choice of $U$. Then $\eta$ is injective and onto. Next we will check that $\eta$ is continuous. For points $z_{i} \in$ $\left[z_{i}^{1} z_{i}^{0}\right], z_{i} \rightarrow y \in\left[y_{1} y_{0}\right]$, since $\left|p y_{0}\right| \leq\left|\uparrow_{y_{1}}^{p} \uparrow_{y_{1}}^{y_{0}}\right| \leq \liminf \left|\uparrow_{z_{i}^{1}}^{p} \uparrow_{z_{i}^{1}}^{z_{0}^{0}}\right|=\liminf \left|p z_{i}^{0}\right|=$ $\left|p y_{0}\right|$, we can get the continuity of $\eta$. On the other hand, we can see that the inverse of $\eta, \bar{\eta}: U \times F \rightarrow g^{-1}(U)$, is defined by $\bar{\eta}\left(\left(x_{0}, f\right)\right)=\left(\left[f_{1} x_{0}\right],\left|f f_{1}\right|\right)$, where $f \in$ $\left[p f_{1}\right]$, and $\left[\uparrow_{f_{1}}^{x_{0}} \uparrow_{f_{1}}^{p}\right]$ is the horizontal lifting of $\left[x_{0} p\right]$. Likewise, we can see that $\bar{\eta}$ is injective, onto and continuous. Hence $\eta$ is a homeomorphism, and obviously $p_{2} \circ \eta=g$, where $p_{2}: U \times F \rightarrow F$ is the projection to the second factor. Thus we get the sublemma.

Thus $X_{1}$ is $D^{J+1}$ - bundle over $S^{I} / \Gamma_{1}$ and $X_{0}$ is $D^{I+1}-$ bundle over $S^{J} / \Gamma_{0}$.

If $I=1$ and $J=1$ (cf. [42, Theorem 14.5]), $\Sigma$ is glued by two solid tori (since by [19] we know that the Klein bottle cannot be embedded into $S^{3}$ ). Hence $\Sigma$ is a Lens space with $\pi_{1}(\Sigma)=\mathbb{Z}_{m}$. Therefore $m=1$.

If $I=1$ and $J>1$, by the long exact sequences:

$$
\begin{gathered}
\rightarrow \pi_{1}\left(S^{J}\right) \rightarrow \pi_{1}\left(\partial X_{1}\right) \rightarrow \pi_{1}\left(S^{1}\right) \rightarrow 0, \\
\rightarrow \pi_{1}\left(S^{1}\right) \rightarrow \pi_{1}\left(\partial X_{0}\right) \rightarrow \pi_{1}\left(S^{J} / \Gamma_{0}\right) \rightarrow 0,
\end{gathered}
$$


we have that $\pi_{1}\left(\partial X_{1}\right)=\mathbb{Z}$ and $\Gamma_{0}$ is a quotient group of $\mathbb{Z}$. Hence $\Gamma_{0}=\mathbb{Z}_{m}$. By Alexander duality, we have that $\mathbb{Z}_{m}=\widetilde{H}_{1}\left(S^{J} / \Gamma_{0} ; \mathbb{Z}\right)=\widetilde{H}_{1}\left(S^{n} \backslash S^{1} ; \mathbb{Z}\right)=$ $\widetilde{H}^{n-1-1}\left(S^{1} ; \mathbb{Z}\right)=0$, where the last identity is because $n>3$. Thus $m=1$. Similarly for $I>1$ and $J=1$.

If $I>1$ and $J>1$, denote $\tilde{X}_{i}=\psi_{i}^{*}\left(X_{i}\right)$, the pull back bundle by the covering map $\psi_{i}$. Then $\tilde{X}_{i}$ is an $m$-fold cover of $X_{i}$. The covering map is denoted by $\phi_{i}, i=0,1$. Since $\pi_{1}\left(\widetilde{X}_{i}\right)=0, \widetilde{X}_{i}$ is the universal cover of $X_{i}$.

Because $\partial \widetilde{X}_{1}$ and $\partial \widetilde{X}_{0}$ are both universal coverings of $\partial X_{1}=\partial X_{0}$, there exists a homeomorphism $h_{0}: \partial \widetilde{X}_{1} \rightarrow \partial \widetilde{X}_{0}$ such that $\phi_{1}=\phi_{0} \circ h_{0}$. Set $\tilde{X}=\tilde{X}_{1} \cup \widetilde{X}_{0} / \sim$ (topologically), where $x \sim h_{0}(x)$ when $x \in \partial \widetilde{X}_{1}$. We will show that $\tilde{X}$ is an $m$-fold cover of $X$.

Let $h: \widetilde{X} \rightarrow X$ be defined by:

$$
h(x)= \begin{cases}\phi_{1}(x), & x \in \widetilde{X}_{1}, \\ \phi_{0}(x), & x \in \widetilde{X}_{0} .\end{cases}
$$

If $x \in \partial \widetilde{X}_{1}, h(x)=\phi_{1}(x)=\phi_{0}\left(h_{0}(x)\right)$. Thus $h$ is well defined and continuous. Next we will check that $h$ is a covering map.

Since for $x \in X_{1} \cap X_{0}$, there exists a small neighborhood $U$ of $x$, denote $U_{i}=$ $X_{i} \cap U$, such that for the closure $\bar{U}_{i}$, we have that $\phi_{i}^{-1}\left(\bar{U}_{i}\right)=\bigcup_{j=1, \cdots, m} \bar{U}_{i j}, i=0,1$, where $\left.\phi_{i}\right|_{\bar{U}_{i j}}$ are homeomorphisms. Thus $h^{-1}(\bar{U})=\bigcup_{j=1, \cdots, m}\left(\bar{U}_{1 j} \cup_{h_{0}} \bar{U}_{0 j^{\prime}}\right)$, and it is clear that $\left.h\right|_{\bar{U}_{1 j} \cup_{h_{0}} \bar{U}_{0 j^{\prime}}}$ is bijective and onto, therefore a homeomorphism (since $\bar{U}_{1 j} \cup_{h_{0}} \bar{U}_{0 j^{\prime}}$ is compact). Thus $\left.h\right|_{U_{1 j} \cup_{h_{0}} U_{0 j^{\prime}}}$ is a homeomorphism. It follows that $\widetilde{X}$ is an $m$-fold cover of $\Sigma$, and therefore $m=1$.

Finally, we will show that $\Sigma=\Sigma_{0} * \Sigma_{1}$. As can be seen in the proof of Lemma 4.7, we have that for $p \in \Sigma, p$ can be uniquely written as $([x y], s)$, where $x \in \Sigma_{1}, y \in \Sigma_{0}$ and $s=|p x|$. By now we can construct a map, $\xi: \Sigma \rightarrow \Sigma_{0} * \Sigma_{1}$, such that $\left.\xi\right|_{\Sigma_{i}}, i=$ 0,1 , are isometries, and maps $u=([x y], s)$ to $([\xi(x) \xi(y)], s)$, where $s=|\xi(u) x|$. For every $p_{1}=\left(\left[x_{1} y_{1}\right], s\right), p_{2}=\left(\left[x_{2} y_{2}\right], t\right) \in \Sigma$, since $\left|x_{1} p_{2}\right|=\left|\xi\left(x_{1}\right) \xi\left(p_{2}\right)\right|$ and $\left|y_{1} p_{2}\right|=\left|\xi\left(y_{1}\right) \xi\left(p_{2}\right)\right|$, by triangle comparison, we get that $\left|p_{1} p_{2}\right| \geq\left|\xi\left(p_{1}\right) \xi\left(p_{2}\right)\right|$. Hence $\angle\left(\uparrow_{p}^{z_{1}}, \uparrow_{p}^{z_{2}}\right) \geq \angle\left(\uparrow_{\xi(p)}^{\xi\left(z_{1}\right)}, \uparrow_{\xi(p)}^{\xi\left(z_{2}\right)}\right)$, for $p \in \Sigma_{1}$ and $z_{i} \in \Sigma$; that is, the induced map, $\xi_{*}: \Sigma_{p} \Sigma \rightarrow \Sigma_{\xi(p)}\left(\Sigma_{0} * \Sigma_{1}\right)$, is 1-Lipschitz. Since $\Sigma_{p} \Sigma$ is isometric to $\Sigma_{\xi(p)}\left(\Sigma_{0} *\right.$ $\left.\Sigma_{1}\right)$, we can see that $\xi_{*}$ is an isometry, especially, $\angle\left(\uparrow_{p}^{p_{1}}, \uparrow_{p}^{p_{2}}\right)=\angle\left(\uparrow \uparrow_{\xi(p)}^{\xi\left(p_{1}\right)}, \uparrow \uparrow_{\xi(p)}^{\xi\left(p_{2}\right)}\right)$. Then by hinge comparison, we have that $\left|p_{1} p_{2}\right| \leq\left|\xi\left(p_{1}\right) \xi\left(p_{2}\right)\right|$. Thus we get that $\xi$ is an isometry.

4.2. Proof of Theorem 4.6. Before we focus on the proof of Theorem 4.6, we collect some lemmas which will be used in the proof of Theorem 4.6. We begin with the following very simple lemma:

Lemma 4.14. Let $X \in \operatorname{Alex}^{n}(\kappa), n \geq 2$, and let $C \subset X$ be a closed locally convex subset with $\operatorname{dim}(C)=n$. If $\partial C \neq \emptyset$, suppose that $C \cap \partial X=\partial C$; then $C=X$.

Proof. First suppose that $\partial C=\emptyset$. We will proceed by induction; if $n=1$, we can see that the lemma holds. For the inductive step, argue by contradiction. Suppose that there is $x \in X \backslash C$; let $y \in C$ be a point such that $|x y|=|x C|$. By induction, we have that $\uparrow_{y}^{x} \in \Sigma_{y} C$. This is a contradiction.

If $\partial C \neq \emptyset$, by considering the double of $X$, we can get the desired result. 
Lemma 4.15. Let the assumptions be as in Theorem 4.6. If $\Sigma_{1}=S^{1}(r)$ with $r \leq 1$, then for any $v \in \Sigma$, there are $v_{i} \in \Sigma_{i}, i=0,1$, such that $v \in\left[v_{0} v_{1}\right]$.

Proof. For $v \in \Sigma$, let $w \in \Sigma_{0}$ be such that $|v w|=\left|v \Sigma_{0}\right|$. Then by Lemma 1.2, we have that $\uparrow_{w}^{v} \in\left(\Sigma_{w} \Sigma_{0}\right)^{\perp}$. Since $\Uparrow_{w}^{S^{1}}=\left(\Sigma_{w} \Sigma_{0}\right)^{\perp}=S^{1}$, the result follows.

Lemma 4.16. Let $A \in \operatorname{Alex}^{n}(1), \partial A \neq \emptyset$. Then $A$ cannot contain a convex closed subset without boundary with positive dimension whose intersection with $\partial A$ is empty.

Proof. Argue by contradiction. Suppose that there exists a convex closed subset $C$ without boundary with positive dimension such that $C \cap \partial A$ is empty. Let $h=\operatorname{dist}_{\partial A}$ and let $p \in C$ be a point such that $h(p)=\min _{x \in C}\{h(x)\}$. By the first variation formula, $\left|\uparrow_{p}^{\partial A} \Sigma_{p} C\right| \geq \frac{\pi}{2}$. By Lemma $1.2,\left|\uparrow_{p}^{\partial A} v\right|=\frac{\pi}{2}$, for any $v \in \Sigma_{p} C$. Then for any $x \in C$, we have that $0=d_{p} h\left(\uparrow_{p}^{x}\right) \geq \frac{h(x)-h(p)}{|p x|} \Rightarrow h(p) \geq h(x)$. Hence $h(x)=$ constant, for any $x \in C$. Let $\gamma \subset C$ be a minimal geodesic. By Theorem 0.5 , there exists a flat rectangle, a contradiction to $A \in \operatorname{Alex}^{n}(1)$.

Lemma 4.17. Let $\Sigma \in \operatorname{Alex}^{n}(1)$. Suppose that $\Sigma$ is homeomorphic to a sphere and topologically nice. Let $\Sigma_{0} \subset \Sigma$ be a convex closed subset with dimension $n-1$, without boundary. Then $\Sigma$ is homeomorphic to $S\left(\Sigma_{0}\right)$, and $\Sigma_{0}$ is homeomorphic to a sphere and topologically nice.

In the proof we will use the following lemma.

Lemma $4.18([24,6.2])$. Let $C \in \operatorname{Alex}^{n}(1)$ with $\partial C \neq \emptyset$. Let $x \in C$ be the unique point with maximal distance from $\partial C$. Then $(C, \partial C) \stackrel{\text { homeo }}{\simeq}\left(\bar{C}\left(\Sigma_{x} C\right), \Sigma_{x} C\right)$, where $\bar{C}\left(\Sigma_{x} C\right) \subset T_{x}$ denotes the closed unit ball at the origin.

Proof of Lemma 4.17. We will prove the lemma by induction on $n$. Clearly when $n=1$ the lemma holds. Suppose the lemma holds for $n-1$.

First apply the inductive assumptions to $\Sigma_{p} \Sigma$ and $\Sigma_{p} \Sigma_{0}$, for $p \in \Sigma_{0}$. We can see that $\Sigma_{0}$ is a topological manifold. Then we have that $\Sigma-\Sigma_{0}$ has two components $H_{1}, H_{2}$, each with set boundary $\Sigma_{0}$. Observe that for every $x, y \in \bar{H}_{i},[x y] \subset \bar{H}_{i}$; if not, by the convexity of $\Sigma_{0}$, we get a contradiction; i.e., $\bar{H}_{i}$ are convex.

By Theorem 2.13, we have that $\partial \bar{H}_{i}=\Sigma_{0}, i=1,2$, and by Lemma 4.18, we have that $\left(\bar{H}_{i}, \partial \bar{H}_{i}\right) \stackrel{\text { homeo }}{\simeq}\left(\bar{C}\left(\Sigma_{v} \Sigma\right), \Sigma_{v} \Sigma\right)$, where $v \in \bar{H}_{i}$ is the point such that $\left|v \partial \bar{H}_{i}\right|=\max \left\{\operatorname{dist}_{\partial \bar{H}_{i}}\right\}$. Hence $\Sigma_{0} \stackrel{\text { homeo }}{\simeq} \Sigma_{v} \Sigma \stackrel{\text { homeo }}{\simeq} S^{n-1}$, where the last one is by the topologically nice property of $\Sigma$. The lemma thus follows.

Lemma 4.19. Let $C \in \operatorname{Alex}^{n}(1)$ with $\partial C=\emptyset$. Let $C_{0} \subset C$ be a convex closed subset with dimension $n-1$ and without boundary. Suppose that $C_{0}$ separates $C$. If there is $v \in C$ such that $\left|v C_{0}\right| \geq \frac{\pi}{2}$, then $\left[v C_{0}\right] \stackrel{\text { isom }}{\cong} S^{+}\left(C_{0}\right)$, where $\left[v C_{0}\right]$ is with the restricted metric. In particular, for $x \in C$ with $|x v|<\frac{\pi}{2}$, we have that $x \in\left[v v_{0}\right]$, for some $v_{0} \in C_{0}$.

Proof. Suppose that $C-C_{0}$ has two components $H_{1}, H_{2}$ and $v \in H_{1} . \bar{H}_{1}$ is convex, as can be seen in the proof of Lemma 4.17. By Theorem 2.13, $\partial \bar{H}_{1}=C_{0}$, and by Lemma 1.2, $|v x|=\frac{\pi}{2}$, for any $x \in C_{0}$. Then $D\left(\bar{H}_{1}\right)=S\left(C_{0}^{\prime}\right)$. By the structure of $D\left(\bar{H}_{1}\right), C_{0}$ separates $D\left(\bar{H}_{1}\right)$, and thus $C_{0}^{\prime}=C_{0}$. For $x \in C$ with $|x v|<\frac{\pi}{2}$, we have that $x \in H_{1}$. The result thus follows. 
Proof of Theorem 4.6. First by Lemma 4.7 and Remark 4.8, we get that $\operatorname{dim}\left(\Sigma_{1}\right) \leq$ 1. By Sublemma 4.9, we have that $\Sigma_{1}$ is convex and isometric to one of the following: $S^{1}(r)$ with $r \leq 1,[a b],\left\{v_{1}, v_{2}\right\}$ with $\left|v_{1} v_{2}\right|=\pi,\{v\}$.

We proceed with the proof by induction. It is easy to see that for $n=2$ the theorem holds. Suppose the theorem holds for $n-1$.

In order to use induction, first we will prove the following:

Sublemma 4.20. For every $p \in \Sigma_{0}$ and every $w \in \Sigma_{p} \Sigma$, we have that $\left|w \Sigma_{p} \Sigma_{0}\right| \leq \frac{\pi}{2}$ and $\left|w\left(\Sigma_{p} \Sigma_{0}\right)^{\perp}\right| \leq \frac{\pi}{2}$.

Proof. Since $\Sigma_{p} \Sigma_{0}$ is convex without boundary in $\Sigma_{p} \Sigma$, by Lemma 1.2 we get that $\left|w \Sigma_{p} \Sigma_{0}\right| \leq \frac{\pi}{2}$. If $w \in \Sigma_{p} \Sigma$ such that $\left|w\left(\Sigma_{p} \Sigma_{0}\right)^{\perp}\right|>\frac{\pi}{2}$, then $\left|w \Uparrow_{p}^{\Sigma_{1}}\right|>\frac{\pi}{2}$. Hence there exists $\uparrow_{p}^{q}$ such that $\left|\uparrow_{p}^{q} \Uparrow_{p}^{\Sigma_{1}}\right|>\frac{\pi}{2}$, and therefore $d_{p} \operatorname{dist}_{\Sigma_{1}}\left(\uparrow_{p}^{q}\right)>0$. Thus there exists $y \in \Sigma$ such that $\left|y \Sigma_{1}\right|>\frac{\pi}{2}$, a contradiction to the assumption of the theorem.

We will prove the inductive steps according to the four situations of $\Sigma_{1}$.

Case 1. Assume $\Sigma_{1}=S^{1}(r)$ with $r \leq 1$. By Lemma 4.15, we can get that $\Sigma$ satisfies the conditions of Theorem 4.4. Then the theorem holds.

Case 2. Assume $\Sigma_{1}=\left\{v_{1}, v_{2}\right\}$ with $\left|v_{1} v_{2}\right|=\pi$. Then $\Sigma=\Sigma_{1} * \Sigma_{1}^{\perp}$. Since $\Sigma$ is topologically nice, $\Sigma_{1}^{\perp}$ is topologically nice and homeomorphic to a sphere. Then by Lemma $4.17, \Sigma_{0}$ is homeomorphic to $S^{n-2}$ and topologically nice.

Case 3. Assume $\Sigma_{1}=\{v\}$.

Subcase 1. Assume $\operatorname{dim}\left(\hat{\Sigma}_{1}\right)=n$. For any $x \in \hat{\Sigma}_{1}$, on one hand, by the assumptions of the theorem, $|x v| \leq \frac{\pi}{2}$; on the other hand, by the definition of $\hat{\Sigma}_{1},|x v| \geq \frac{\pi}{2}$. Thus $|x v|=\frac{\pi}{2}$. By Lemma 4.7, we get a contradiction.

Subcase 2. Assume $\operatorname{dim}\left(\hat{\Sigma}_{1}\right)=n-1$. Subsubcase 1. Assume $\partial \hat{\Sigma}_{1}=\emptyset$. On one hand, by Lemma 4.17, $\Sigma$ is homeomorphic to $S\left(\hat{\Sigma}_{1}\right)$; thus $\hat{\Sigma}_{1}$ separates $\Sigma$. On the other hand, for every $w \in \Sigma-\left(\hat{\Sigma}_{1} \cup \Sigma_{1}\right)$, we have that $\left|w \hat{\Sigma}_{1}\right|<\frac{\pi}{2}$ and $\left|w \Sigma_{1}\right|<\frac{\pi}{2}$. Then by triangle comparison, we get that dist $_{\hat{\Sigma}_{1}}$ is noncritical in $\Sigma-\left(\hat{\Sigma}_{1} \cup \Sigma_{1}\right)$; thus there is a deformation retraction from $\Sigma-\hat{\Sigma}_{1}$ to $\{v\}$, a contradiction.

Subcase 3. Assume $\partial \hat{\Sigma}_{1} \neq \emptyset$. We first show that for any $x \in\left(\hat{\Sigma}_{1}\right)^{\circ}=\left(\hat{\Sigma}_{1}-\partial \hat{\Sigma}_{1}\right)$, $\#\left\{\Uparrow_{x}^{v}\right\}=2$. By Lemma 4.17, $\Sigma_{x} \Sigma \stackrel{\text { homeo }}{\simeq} S\left(\Sigma_{x} \hat{\Sigma}_{1}\right)$, and by the proof of Lemma 4.19, we have that $\#\left\{\Uparrow_{x}^{v}\right\} \leq 2$. Suppose $\#\left\{\Uparrow_{x}^{v}\right\}=1$. Then by Lemma 4.19, there is $w \in \Sigma_{x} \Sigma$, such that $d_{x} \operatorname{dist}_{v}(w)>0$, a contradiction, as can be seen in the proof of Sublemma 4.20.

By Lemma 4.16 , we have that $\Sigma_{0} \cap \partial \hat{\Sigma}_{1} \neq \emptyset$. If $\Sigma_{0} \cap \partial \hat{\Sigma}_{1}$ is not equal to $\Sigma_{0}$, i.e., there is $z \in \Sigma_{0} \cap\left(\hat{\Sigma}_{1}\right)^{\circ}$, then by the above paragraph, we have that $\#\left\{\Uparrow_{z}^{v}\right\}=2$. Thus for any $x \in \Sigma_{0}$, \#\{个r $\left.\uparrow_{x}^{v}\right\}=2$, because by induction and the proof of Sublemma 4.11, we have that for any $p, q \in \Sigma_{0}$, \#\{ $\left\{\Uparrow_{p}^{v}\right\}=\#\left\{\Uparrow_{q}^{v}\right\}$. By the proof of Sublemma 4.12, we can see that $\psi: \Uparrow_{v}^{\hat{\Sigma}_{1}^{\circ}} \rightarrow \hat{\Sigma}_{1}^{\circ}, \psi(\Uparrow y)=y, y \in \hat{\Sigma}_{1}^{\circ}$, is a locally isometric covering map. Since $\hat{\Sigma}_{1}$ is contractible (the reason is that $\hat{\Sigma}_{1} \in \operatorname{Alex}^{n-1}(1)$, with nonempty boundary; hence the soul of $\hat{\Sigma}_{1}$ is a point (cf. [24])), $\Uparrow_{v}^{\hat{\Sigma}_{1}^{\circ}} \subset \Sigma_{v} \Sigma$ are two copies of $\hat{\Sigma}_{1}^{\circ}$; by taking closure we can see that $\Uparrow_{v}^{\Sigma_{0}}$ are two copies of $\Sigma_{0}$ with empty intersection. This is impossible, since by applying the inductive assumptions to $\Sigma_{p} \Sigma$ and $\Sigma_{p} \Sigma_{0}$, for $p \in \Sigma_{0}$, we can see that $\Sigma_{0}$ is a topological manifold, and one component of $\Uparrow_{v}^{\Sigma_{0}}$ 
separates $\Sigma_{v}$, which is homeomorphic to a sphere, into two components, with the closure of each one convex. By Lemma 4.16, we get a contradiction.

It follows that for any $p \in \Sigma_{0}, \#\left\{\Uparrow_{p}^{v}\right\}=1$. Hence $\Sigma_{0} \subset \partial \hat{\Sigma}_{1}$. We claim that $\partial \hat{\Sigma}_{1} \stackrel{\text { homeo }}{\simeq} S^{n-1}$. Therefore $\Sigma_{0}=\partial \hat{\Sigma}_{1}$. It follows that $\Sigma$ contains two copies of $S^{+}\left(\hat{\Sigma}_{1}\right)$, with each copy convex gluing along the boundary, which is homeomorphic to $S^{n}$. Thus $\Sigma=\left[v \hat{\Sigma}_{1}\right]$, a double of $v * \hat{\Sigma}_{1}$.

We now verify the claim: Let $s \in \hat{\Sigma}_{1}$ be a point such that $\left|s \partial \hat{\Sigma}_{1}\right|=\max \left\{\operatorname{dist}_{\partial \hat{\Sigma}_{1}}\right\}$. By Lemma 4.17, $\Sigma_{s} \Sigma \stackrel{\text { homeo }}{\simeq} S\left(\Sigma_{s} \hat{\Sigma}_{1}\right)$ and $\Sigma_{s} \hat{\Sigma}_{1} \stackrel{\text { homeo }}{\simeq} S^{n-1}$. By Lemma 4.18, $\left(\hat{\Sigma}_{1}, \partial \hat{\Sigma}_{1}\right) \stackrel{\text { homeo }}{\simeq}\left(\bar{C}\left(\Sigma_{s} \hat{\Sigma}_{1}\right), \Sigma_{s} \hat{\Sigma}_{1}\right)$. Hence $\partial \hat{\Sigma}_{1} \stackrel{\text { homeo }}{\simeq} S^{n-1}$.

Subcase 4. Assume $\operatorname{dim}\left(\hat{\Sigma}_{1}\right)=n-2$. By Lemma 4.14, we get that $\hat{\Sigma}_{1}=\Sigma_{0}$. Hence for every $w \in \Sigma-\left(\Sigma_{0} \cup \Sigma_{1}\right),\left|w \Sigma_{0}\right|<\frac{\pi}{2},\left|w \Sigma_{1}\right|<\frac{\pi}{2}$. By triangle comparison, we get that dist $_{\Sigma_{i}}$ is noncritical in $\Sigma-\left(\Sigma_{0} \cup \Sigma_{1}\right)$. Therefore there are deformation retractions from $\Sigma-\Sigma_{1}$ to $\Sigma_{0}$, and from $\Sigma-\Sigma_{0}$ to $\Sigma_{1}$. Since for $p \in \Sigma_{0}$, by induction, $\Sigma_{p} \Sigma_{0}$ is homeomorphic to a sphere, $\Sigma_{0}$ is thus a topological manifold. As in Theorem 4.4, by using Alexander duality, we get a contradiction.

Case 4. Assume $\Sigma_{1}=[a b]$.

Subcase 1. Assume $\operatorname{dim}\left(\hat{\Sigma}_{1}\right)=n$. Similarly as in Subcase 1 of Case 3, we get a contradiction.

Subcase 2. Assume $\operatorname{dim}\left(\hat{\Sigma}_{1}\right)=n-1$. First by Lemma 4.7, we have that $\partial \hat{\Sigma}_{1} \neq \emptyset$. By Remark 1.3, we have that if there exist $x \in[a b]^{\circ}$ and $y \in\left(\hat{\Sigma}_{1}\right)^{\circ}$, such that $|x y|=\frac{\pi}{2}$, then for every $\bar{x} \in[a b]^{\circ}$ and every $\bar{y} \in \hat{\Sigma}_{1},|\bar{x} \bar{y}|=\frac{\pi}{2}$. By Lemma 4.7, we get a contradiction. Hence for every $x \in[a b]^{\circ}$ and every $y \in\left(\hat{\Sigma}_{1}\right)^{\circ},|x y|>\frac{\pi}{2}$. Since by Lemma 1.2, we have that for every $x \in[a b]$ and for every $z \in \Sigma_{0},|x z|=\frac{\pi}{2}$, it follows that $\Sigma_{0} \subset \partial \hat{\Sigma}_{1}$.

Let $s \in \hat{\Sigma}_{1}$ be the point such that $\left|s \partial \hat{\Sigma}_{1}\right|=\max \left\{\operatorname{dist}_{\partial \hat{\Sigma}_{1}}\right\}$. By Lemma 4.17, $\Sigma_{s} \Sigma \stackrel{\text { homeo }}{\simeq} S\left(\Sigma_{s} \hat{\Sigma}_{1}\right)$ and $\Sigma_{s} \hat{\Sigma}_{1} \stackrel{\text { homeo }}{\simeq} S^{n-2}$, and by Lemma 4.18, $\left(\hat{\Sigma}_{1}, \partial \hat{\Sigma}_{1}\right) \stackrel{\text { homeo }}{\simeq}$ $\left(\bar{C}\left(\Sigma_{s} \hat{\Sigma}_{1}\right), \Sigma_{s} \hat{\Sigma}_{1}\right)$. Hence $\partial \hat{\Sigma}_{1} \stackrel{\text { homeo }}{\simeq} S^{n-2}$. On the other hand, by applying the inductive assumptions to $\Sigma_{p} \Sigma$ and $\Sigma_{p} \Sigma_{0}$, for $p \in \Sigma_{0}$, we can see that $\Sigma_{0}$ is a topological manifold. Hence $\Sigma_{0}=\partial \hat{\Sigma}_{1}$.

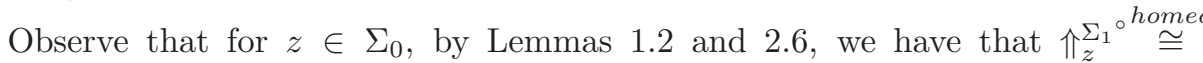
$(a, b) \times \Uparrow_{z}^{x}$, where $x \in[a b]^{\circ}$. Since $\Uparrow_{z}^{\Sigma_{1}} \subset\left(\Sigma_{z} \Sigma_{0}\right)^{\perp}$, whose dimension (by Lemma $4.7) \leq 1$, we have that $\#\{[x z]\}$ is finite. By the proof of Sublemma 4.12, we can see that $\psi_{0}: \Uparrow_{x}^{\Sigma_{0}} \rightarrow \Sigma_{0}, \psi_{0}\left(\Uparrow_{x}^{y}\right)=y, y \in \Sigma_{0}$, is a locally isometric covering map. Since $\Sigma_{0} \stackrel{\text { homeo }}{\simeq} S^{n-2}, \Uparrow_{x}^{\Sigma_{0}}$ are several copies of $\Sigma_{0}$. On the other hand, suppose that $\Sigma_{x} \Sigma=S\left(\Sigma^{\prime}\right)$; then $\Sigma^{\prime}$ is homeomorphic to $S^{n-2}$ (by the topologically nice property of $\Sigma$ ). Hence $\Sigma^{\prime}=\Uparrow_{x}^{\Sigma_{0}} \stackrel{\text { isom }}{\cong} \Sigma_{0}$, and $\#\{[x z]\}=1$. By now we get that $\left[\Sigma_{0} \Sigma_{1}\right]=\Sigma_{0} * \Sigma_{1}$, which can be seen in the final part of the proof of Theorem 4.4.

Finally, we need to show that for every $x \in\left(\hat{\Sigma}_{1}\right)^{\circ},|a x|=\frac{\pi}{2}$ and $|b x|=\frac{\pi}{2}$. Since by the construction of $\hat{\Sigma}_{1}$ and by the condition of the theorem, $|x,[a b]|=\frac{\pi}{2}$, we have showed that $\left|x,[a b]^{\circ}\right|>\frac{\pi}{2}$; thus $|a x|=\frac{\pi}{2}$ or $|b x|=\frac{\pi}{2}$. Without loss of generality, suppose that $|a x|=\frac{\pi}{2}$. We have that $\#\{[a x]\}=1$. If not, as in Subsubcase 2 in Case 3, we get that $\Sigma=D\left(S^{+}\left(\hat{\Sigma}_{1}\right)\right)$, a contradiction. Then by Lemma 4.17 and 
Lemma 4.19, $\left|\uparrow_{x}^{a} \uparrow_{x}^{b}\right|>\frac{\pi}{2}$. It follows that $d_{x} \operatorname{dist}_{\Sigma_{1}}\left(\uparrow_{x}^{b}\right)>0$. This contradicts the condition of the theorem, as can be seen in the proof of Sublemma 4.20.

Thus $\Sigma$ contains $\left[\Sigma_{0} \Sigma_{1}\right]$ and two copies of $S^{+}\left(\hat{\Sigma}_{1}\right)$, gluing along two copies of $S^{+}\left(\Sigma_{0}\right)$, which is homeomorphic to $S^{n}$. Hence $\Sigma=\left(a * \hat{\Sigma}_{1} \cup_{\hat{\Sigma}_{1}} b * \hat{\Sigma}_{1}\right) \cup_{\partial}\left(\Sigma_{1} * \Sigma_{0}\right)$.

Subcase 3. Assume $\operatorname{dim}\left(\hat{\Sigma}_{1}\right)=n-2$. Similarly as in the proof of Subcase 3 of Case 3 , we get a contradiction.

Now we are ready to verify Proposition 2.1.

Proof of Proposition 2.1. Observe that for any $p \in S$, we have that $\Uparrow_{p}^{\partial \Omega_{c}} \subset\left(\Sigma_{p} S\right)^{\perp}$, and by applying the first variation formula to the Busemann function, we get that for all $v \in \Sigma_{p} A,\left|v \Uparrow_{p}^{\partial \Omega_{c}}\right| \leq \frac{\pi}{2}$. Hence $\left|v\left(\Sigma_{p} S\right)^{\perp}\right| \leq \frac{\pi}{2}$. Thus for $p \in A, \Sigma_{p} A$ satisfies the conditions of Theorem 4.6. Therefore (2.1.1), (2.1.2) and (2.1.3) can all be derived from Theorem 4.6.

(2.1.4): As can be seen from the proof of Theorem 4.6, for any $x \in[a b]^{\circ}$, there is $y \in \hat{\Sigma}_{1}^{p}$ such that $|x y|>\frac{\pi}{2}$. Then either $a$ or $b$ must be in $E$. If $b \notin E$, then also from the proof, there exists a point with distance bigger than $\frac{\pi}{2}$ to $a$. The first statement thus follows. The second statement is easily seen.

\section{ACKNOWLEDGEMENTS}

First the author sincerely thanks Professor Xiaochun Rong for many useful discussions during the preparation of this work, especially for his vast time spent on the checking, organization and presentation of this work. Second, the author owes his thanks to Professor Jianguo Cao for his instructive work. The author is also in great debt to Professor Xuezhi Zhao for his unselfishness on topology and many useful conversations. Furthermore, the author would like to thank Postdoc Shicheng $\mathrm{Xu}$ for many constructive comments and useful discussions. Finally, the author would like to thank Yusheng Wang, Xiaole Su, Hongwei Sun and Chao Qian for their useful comments.

\section{REFERENCES}

[1] S. Alexander, V. Kapovitch and A. Petrunin, Alexandrov's geometry, in preparation.

[2] Dmitri Burago, Yuri Burago, and Sergei Ivanov, A course in metric geometry, Graduate Studies in Mathematics, vol. 33, American Mathematical Society, Providence, RI, 2001. MR.1835418(2002e:53053)

[3] Yu. Burago, M. Gromov, and G. Perel'man, A. D. Aleksandrov spaces with curvatures bounded below (Russian, with Russian summary), Uspekhi Mat. Nauk 47 (1992), no. 2(284), 3-51, 222, DOI 10.1070/RM1992v047n02ABEH000877; English transl., Russian Math. Surveys 47 (1992), no. 2, 1-58. MR1185284 (93m:53035)

[4] Russell G. Brasher, A separation theorem for manifolds, Proc. Amer. Math. Soc. 23 (1969), 242-245. MR0250313(40 \#3552)

[5] L. E. J. Brouwer, Beweis des Jordanschen Satzes für den n-dimensionalen Raum (German), Math. Ann. 71 (1911), no. 3, 314-319, DOI 10.1007/BF01456847. MR:1511659

[6] Jeff Cheeger and David G. Ebin, Comparison theorems in Riemannian geometry, NorthHolland Mathematical Library, Vol. 9, North-Holland Publishing Co., Amsterdam, 1975. MR0458335 (56 \#16538)

[7] J. Cao, B. Dai and J. Mei, An optimal extension of Perelman's comparison theorem for quadrangles and its applications, In book "Recent Advances in Geometric Analysis", edited by Yng-Ing Lee, Chang-Shou Lin, Mao-Pei Tsui. Advanced Lectures in Mathematics, Vol. 11, ISBN: 978-7-01-027602-2, 229 pages, Higher Educational Press and Internationaal Press, Beijing and Boston, 2009-2010. MR2648938(2011d:53074) 
[8] J. Cao, B. Dai and J. Mei, An extension of Perelman's soul theorem for singular spaces, arXiv: 0706.0565.

[9] Jianguo Cao, Bo Dai, and Jiaqiang Mei, A quadrangle comparison theorem and its application to soul theory for Alexandrov spaces, Front. Math. China 6 (2011), no. 1, 35-48, DOI 10.1007/s11464-010-0079-4. MR 2762970(2011m:53050)

[10] Jeff Cheeger and Detlef Gromoll, On the structure of complete manifolds of nonnegative curvature, Ann. of Math. (2) 96 (1972), 413-443. MR0309010 (46 \#8121)

[11] Robert D. Edwards and Robion C. Kirby, Deformations of spaces of imbeddings, Ann. Math. (2) 93 (1971), 63-88. MR0283802 (44 \#1032)

[12] Jianguo Cao and Mei-Chi Shaw, The smoothness of Riemannian submersions with nonnegative sectional curvature, Commun. Contemp. Math. 7 (2005), no. 1, 137-144, DOI 10.1142/S0219199705001672. MR2129792 (2006g:53035)

[13] Michael H. Freedman and Frank Quinn, Topology of 4-manifolds, Princeton Mathematical Series, vol. 39, Princeton University Press, Princeton, NJ, 1990. MR1201584 (94b:57021)

[14] Karsten Grove and Steen Markvorsen, New extremal problems for the Riemannian recognition program via Alexandrov geometry, J. Amer. Math. Soc. 8 (1995), no. 1, 1-28, DOI 10.2307/2152882. MR 1276824 (95j:53066)

[15] Detlef Gromoll and Wolfgang Meyer, On complete open manifolds of positive curvature, Ann. of Math. (2) 90 (1969), 75-90. MR0247590 (40 \#854)

[16] Detlef Gromoll and Kristopher Tapp, Nonnegatively curved metrics on $S^{2} \times \mathbb{R}^{2}$, Geom. Dedicata 99 (2003), 127-136, DOI 10.1023/A:1024967423590. MR.1998931(2004m:53061)

[17] Luis Guijarro, Improving the metric in an open manifold with nonnegative curvature, Proc. Amer. Math. Soc. 126 (1998), no. 5, 1541-1545, DOI 10.1090/S0002-9939-98-04287-7. MR:1443388 (98j:53042)

[18] Luis Guijarro and Gerard Walschap, The metric projection onto the soul, Trans. Amer. Math. Soc. 352 (2000), no. 1, 55-69, DOI 10.1090/S0002-9947-99-02237-0. MR 1487617 (2000c:53034)

[19] Allen Hatcher, Algebraic topology, Cambridge University Press, Cambridge, 2002. MR:1867354(2002k:55001)

[20] Vitali Kapovitch, Perelman's stability theorem, Surveys in differential geometry. Vol. XI, Surv. Differ. Geom., vol. 11, Int. Press, Somerville, MA, 2007, pp. 103-136. MR2408265 (2009g:53057)

[21] V. Kapovitch, Regularity of limits of noncollapsing sequences of manifolds, Geom. Funct. Anal. 12 (2002), no. 1, 121-137, DOI 10.1007/s00039-002-8240-1. MR1904560(2003b:53043)

[22] Vitali Kapovitch, Anton Petrunin, and Wilderich Tuschmann, Nilpotency, almost nonnegative curvature, and the gradient flow on Alexandrov spaces, Ann. of Math. (2) 171 (2010), no. 1, 343-373, DOI 10.4007/annals.2010.171.343. MR2630041(2011d:53063)

[23] A. D. Milka, Metric structure of a certain class of spaces that contain straight lines (Russian), Ukrain. Geometr. Sb. Vyp. 4 (1967), 43-48. MR0256327(41 \#983)

[24] G. Perelman, Alexandrov's spaces with curvature bounded from below II (preprint, 1991; see http://www.math.psu.edu/petrunin/papers/papers.html).

[25] G. Ya. Perel'man, Elements of Morse theory on Aleksandrov spaces (Russian, with Russian summary), Algebra i Analiz 5 (1993), no. 1, 232-241; English transl., St. Petersburg Math. J. 5 (1994), no. 1, 205-213. MR1220498(94h:53054)

[26] G. Perelman, Proof of the soul conjecture of Cheeger and Gromoll, J. Differential Geom. 40 (1994), no. 1, 209-212. MR.1285534(95d:53037)

[27] G. Perelman and A. Petrunin, Quasi-geodesics and gradient curves in Alexandrov spaces (preprint, 1996; see http://www.math.psu.edu/petrunin/papers/papers.html).

[28] Peter Petersen, Riemannian geometry, Graduate Texts in Mathematics, vol. 171, SpringerVerlag, New York, 1998. MR1480173 (98m:53001)

[29] A. Petrunin, Parallel transportation for Alexandrov space with curvature bounded below, Geom. Funct. Anal. 8 (1998), no. 1, 123-148, DOI 10.1007/s000390050050. MR1601854 (98j:53048)

[30] Anton Petrunin, Semiconcave functions in Alexandrov's geometry, Surveys in differential geometry. Vol. XI, Surv. Differ. Geom., vol. 11, Int. Press, Somerville, MA, 2007, pp. 137201. MR2408266 (2010a:53052)

[31] Conrad Plaut, Metric spaces of curvature $\geq k$, Handbook of geometric topology, NorthHolland, Amsterdam, 2002, pp. 819-898. MR 1886682 (2002m:53063) 
[32] Xiaochun Rong and Shicheng Xu, Stability of $e^{\epsilon}$-Lipschitz and co-Lipschitz maps in GromovHausdorff topology, Adv. Math. 231 (2012), no. 2, 774-797, DOI 10.1016/j.aim.2012.05.018. MR2955191

[33] Stephen B. Seidman, Completely regular mappings with locally compact fiber, Trans. Amer. Math. Soc. 147 (1970), 461-471. MR0254819 (40 \#8026)

[34] V. A. Šarafutdinov, The Pogorelov-Klingenberg theorem for manifolds that are homeomorphic to $\mathbf{R}^{n}$ (Russian), Sibirsk. Mat. Ž. 18 (1977), no. 4, 915-925, 958. MR0487896 (58 \#7488)

[35] Katsuhiro Shiohama, An introduction to the geometry of Alexandrov spaces, Lecture Notes Series, vol. 8, Seoul National University Research Institute of Mathematics Global Analysis Research Center, Seoul, 1993. MR1320267(96c:53064)

[36] Norman Steenrod, The Topology of Fibre Bundles, Princeton Mathematical Series, vol. 14, Princeton University Press, Princeton, N. J., 1951. MR0039258 (12,522b)

[37] Takashi Shioya and Takao Yamaguchi, Collapsing three-manifolds under a lower curvature bound, J. Differential Geom. 56 (2000), no. 1, 1-66. MR1863020 (2002k:53074)

[38] Gerard Walschap, Nonnegatively curved manifolds with souls of codimension 2, J. Differential Geom. 27 (1988), no. 3, 525-537. MR940117 (89g:53067)

[39] Burkhard Wilking, A duality theorem for Riemannian foliations in nonnegative sectional curvature, Geom. Funct. Anal. 17 (2007), no. 4, 1297-1320, DOI 10.1007/s00039-007-0620-0. MR2373019 (2009a:53047)

[40] Andreas Wörner, Boundary strata of nonnegatively curved Alexandrov spaces and a splitting theorem, Ph.D. thesis, der Westfälischen Wilhelms-Universität Münster, 2010.

[41] Jyh-Yang Wu, Topological regularity theorems for Alexandrov spaces, J. Math. Soc. Japan 49 (1997), no. 4, 741-757, DOI 10.2969/jmsj/04940741. MR1466363(98h:53071)

[42] T. Yamaguchi, Collapsing 4-manifolds under a lower curvature bound, preprint, 2002.

Department of Mathematics, Capital Normal University, Beijing, People's Republic OF CHINA

E-mail address: xuepingli@jsnu.edu.cn 